\title{
HINRL4Rec: A Novel Integrated Heterogeneous Network Embedding with Reinforcement Learning for Recommendation
}

Tham Vo ( $\sim$ thamvth@tdmu.edu.vn )

Thu Dau Mot University https://orcid.org/0000-0001-7291-4168

\section{Research Article}

Keywords: HIN, BERT, network embedding, reinforcement learning, recommendation.

Posted Date: September 9th, 2021

DOl: https://doi.org/10.21203/rs.3.rs-610194/v1

License: (c) (i) This work is licensed under a Creative Commons Attribution 4.0 International License.

Read Full License 


\title{
HINRL4Rec: a novel integrated heterogeneous network embedding with reinforcement learning for recommendation
}

\author{
Tham $\mathrm{Vo}^{1, *}$ \\ ${ }^{I}$ Thu Dau Mot University, Binh Duong, Vietnam \\ ${ }^{1}$ thamvth@tdmu.edu.vn \\ ${ }^{(*)}$ Corresponding author
}

\begin{abstract}
Recent KG-oriented recommendation techniques mainly focus on the direct interaction between entities in the given KGs as the rich information sources for leveraging the quality of recommendation outputs. However, they are still hindered by the heterogeneity, type-varied entities and their relationships in knowledge graphs (KG) as the heterogeneous information networks (HIN). This limitation seems challenging to build up an effective approach for the KG-based recommendation system in both semantic path-based exploitation and heterogeneous information extraction. To meet these challenges, we proposed a novel integrated HIN embedding with reinforcement learning ( $\underline{\mathrm{RL}})$-based feature engineering for recommendation, called as: HINRL4Rec. First of all, we apply the combined textual meta-path-based embedding approach for learning multiple-rich-schematic representations of user/item and their associated entities. Then, these extracted multi-typed embeddings of user and item entities are fused into the unified embedding spaces during the KG embedding process. Finally, the unified representations of users and items are then used to facilitate the RL-based policy-driven searching process in the next steps for performing the recommendation task. Extensive experiments in real-world datasets demonstrate the effectiveness of our proposed model in comparing with recent state-of-the-art recommendation baselines.
\end{abstract}

Keywords: HIN; BERT; network embedding; reinforcement learning; recommendation.

\section{INTRODUCTION}

Recently, recommendation system [1] has become a standard application for multiple platforms, such as ecommerce (Amazon, Alibaba, etc.), social networks (Facebook, Twitter, etc.), content-based sharing network (YouTube, TikTok, etc.). The classical recommendation approaches, like as: matrix factorization (MF) or factorization machine (FM) mainly rely on the direct user-item preferences to facilitate the recommendation task. However, these traditional techniques encounter limitations related to the sparsity of user-item interaction data as well as integrating with the auxiliary information from external resources like as user-item associated network's structures or external knowledge graphs (KG). In fact, associating the designed recommendation models with the capability to integrate with external resources, like as: KGbased data, information networked data, textual data, etc. not only enables our recommender system to achieve better performance in exploitation of various unstructured/semi-structured/structured information but also equips the designed recommendation models with the capability of handling explainable recommendation tasks. The explainable recommendation has recently task become popular and is widely studied by researchers due to its capability of intuitively understanding complex user-item preferences as well as flexibly integrating with inter-connecting relationships with other nodes/entities in the context of heterogeneous information networks. In recent years, researchers have discovered the potential applications of integrating external heterogeneous networks as the enriched "side information" such as social networked data, knowledge graph in improving the performance of personalized recommendation task by taking advances of automatic graph-based feature engineering via network embedding techniques. In fact, realworld heterogeneous networks, such as social networks, knowledge graphs, etc. include multi-typed nodes/entities and links/relationships which are considered as the powerful information modelling 
approaches for exploiting the rich semantic information of user-item preferences. These rich semantic resources have been studied and verified capabilities of enhancing the performance in recommendation task. The basic principle of HIN-based recommendation is to use the extracted latent embedding features from the associated networks to facilitate the process of analyzing user-item relatedness and formulate the recommendation scenarios via different techniques such as matrix factorization, like as: HERec [1], HetNERec [2], etc. or reinforcement learning searching strategy, like as: PGPR [3], SCPR [4], etc. Recently, information extraction from knowledge graphs is widely researched by researchers and applied in multiple domains due to the popularity and freely availability of real-world KGs (Wikipedia, Freebase, Yelp, etc.). The extracted information from the current KGs can be considered as valuable resources for welly understanding the user-item relationships as well as explainable path-based recommendation reasoning processes. To properly extract knowledge from KGs, multiple KG embedding (a.k.a., representation learning) techniques have been proposed to tackle the entity/link vector-based transformation process. Trans-family models (TransE [5], TransH [6], TransR [7], etc.) are considered as the most well-known KG embedding techniques which enable to effectively transform the entities and relationships of a given $\mathrm{KG}$ into regularized continuous vectors and simultaneously uncover the proximity between KG's entities by analyzing their representation relatedness via different direct relations. Taking $\mathrm{KG}$ embedding as a general network embedding task, multiple network-based embedding techniques such as: DeepWalk [8], LINE [9] and Node2Vec [10], etc. also can be applied to learn the rich-semantic latent representations of entities in knowledge graphs.

\subsection{Existing challenges \& motivations}

As mentioned above, recent studies have demonstrated great achievements in using KG/network embedding approach can help to leverage the accuracy performance of recommendation task. However, these previous $\mathrm{KG} /$ network embedding approaches are considered as lacking capability to learn the long-range dependency multi-hop relationships between entities in a given $\mathrm{KG}$, such as: Trans-family models [5] [6] [7] or Node2Vec [10] majorly relies on the direct relationships between pairwise entities, e.g., user_1 $\stackrel{\text { like }}{\longrightarrow}$ product_1, user_2 $\stackrel{\text { friend }}{\longrightarrow}$ user_1, etc. They might fail to capture the explainable long-range dependency path like as: user_2 $\stackrel{\text { friend }}{\longrightarrow}$ user_1 $\stackrel{\text { like }}{\longrightarrow}$ product_1 which indicates the indirect relatedness of [user_2] and [product_1] and should be potentially recommended for he/she. In the context of heterogeneous networks, natural multi-typed entities and relationships in a given KG might carry different semantic meanings which are normally represented as multi-hop links, can be formulated as the "meta-paths" [11] [12], such as: $\mathrm{P}$ [product $] \stackrel{\text { belong }}{\longrightarrow} \mathrm{C}[$ category $] \stackrel{\text { belong }}{\longleftarrow} \mathrm{P}$ and $\mathrm{P} \stackrel{\text { belong }}{\longrightarrow} \mathrm{B}[$ Brand $] \stackrel{\text { belong }}{\longleftarrow} \mathrm{P}$ indicate the same-category and same-brand products semantic relationships, respectively. These semantic representations might be valuable for recommendation scenario where they demonstrate the relatedness of shared preferences of users who might have the same interests on same-category and same-brand product sets. Recent well-known studies in HIN embedding-based for recommendation, such as HERec [1] and HetNERec [2] models propose a novel utilization of meta-path-based network embedding technique to properly encode the KG's entities via different semantic paths for enriching the user-item preferences in the context of heterogeneous network which are then used to facilitated the matrix factorization mechanism to improve the performance of recommendation. However, these HIN embedding-based model still mostly depend on the matrix factorization process for the fixed sets of user-item recommendation decisions and unable to explain the recommendation results in the context of latent representation learning for networked data. Moreover, recent recommendation techniques also lack of thorough considerations on evaluating the semantic textual information of target-typed entities for recommendation task (e.g., users and products/items) in given KGs

To deal with challenges of unexplainable MF-based recommendation approach, path-based reasoning recommendation models [13] [3] [4] [14] have been proposed recently. Most of recent path-based reasoning methods for recommendation, such as PGPR [3], SCPR [4] and KGPolicy [14] mainly focus on learning the direct relatedness representations of KG's entities to facilitate the policy-oriented reasoning process for personalized recommendation task via reinforcement learning (RL) approach. By taking advantages of 
reinforcement learning policy-guided searching strategy, the proposed RL-based methods can conduct the flexible and explainable user-item relationship recommendation by utilizing the of preferred attributes of user-item relatedness as the interpretable paths. However, these existing RL-based methods only concentrate on utilizing the direct-order proximity attributes between KG's entities and apply direct-relation embedding technique to implicitly mapping these attributes into the latent vector spaces which is considered as unable to fully learn all rich-semantic inter-connecting relations between entities in forms of meta-paths. Moreover, even these path-driven reasoning techniques can effectively produce the explainable recommendation results following defined semantic paths between user-item pairs by they might fail to consistently learn the similarity matching between embedding vectors of users and items. Hence, these models are considered as unable to capture the semantic long-range dependent relationships between users and items in context of heterogeneity.

\subsection{Our contributions}
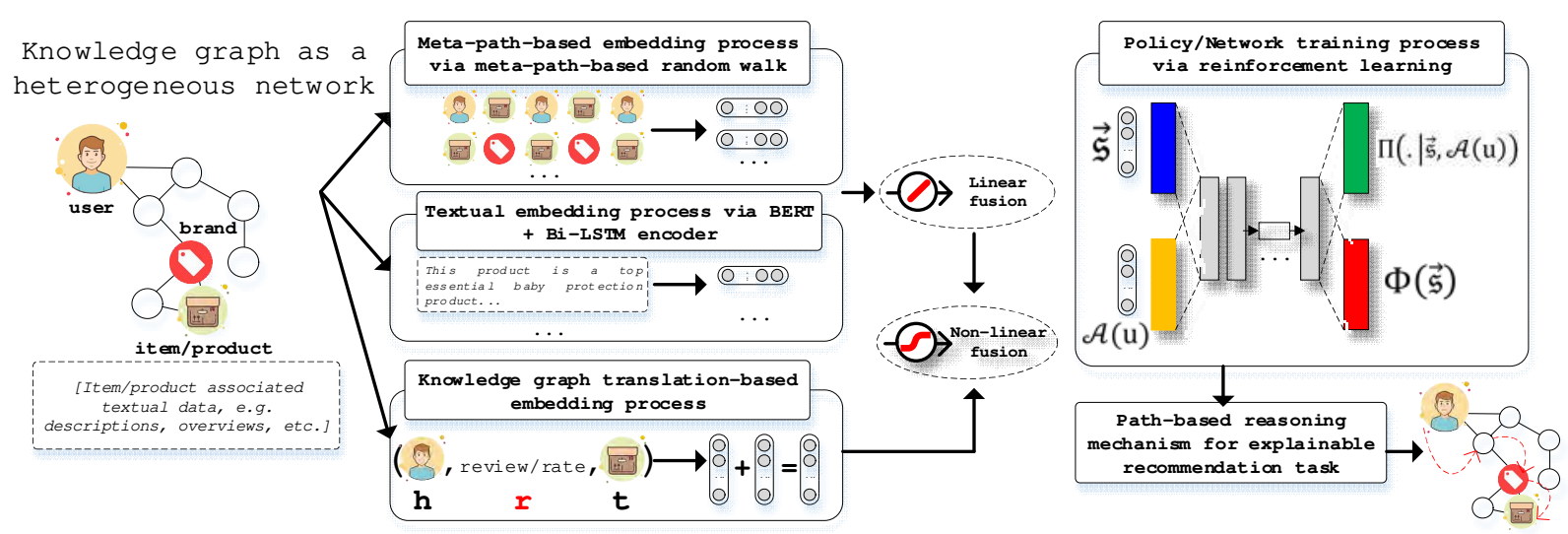

Figure 1. The illustration of overall architecture of our proposed HINRLARec model in this paper

To deal with above listed limitations, in this paper we propose a novel approach of integrated HIN-based embedding with the policy-oriented searching strategy via reinforcement learning ( $\underline{\mathrm{RL}})$ approach for handling explainable personalized recommendation task, called as: HINRE4Rec. First of all, to effectively learn the rich contextual latent representations of user and item entities in a given KG via different semantic inter-connecting relationships in forms of meta-paths, we apply the meta-path-based embedding technique to learn the path-varied representations of users and items. Moreover, to take advantages and effectively capturing the rich semantic representations of textual data which is associated to target-typed entities, we apply a combined pre-trained BERT [15] and Bi-LSTM encoding mechanism. Then, the achieved rich textual semantic representations of target-typed entities are merged into the meta-path-based representations via a linear fusion layer to enrich the latent embedding features of given entities.

Then, from the extracted rich semantic meta-path-varied representations of user and item entities, we apply a personalized non-linear fusion mechanism to merge these meta-path-varied representations into the unified embedding spaces which are then used to enrich the recommendation-oriented KG embedding process via the TransE algorithm. The parameters of our defined fusion function are jointly optimized during the $\mathrm{KG}$ embedding process.

Finally, the achieved final latent representations of multi-typed entities in a given $\mathrm{KG}$, are then used to facilitate the policy-oriented path-based searching strategy to sample explainable user-item interconnecting paths for fulfilling the personalized recommendation task during inference process via the RL. Figure 1 illustrates the overall architecture of our proposed HINRL4Rec model in this paper. In general, our contributions in this paper can be summarized as threefold, as following:

- First, we shortly describe the importance of utilizing external heterogeneous information networks, e.g., KG, social networked data, etc. as side information for leveraging the performance of 
personalized recommendation task. To effectively integrate the HIN-based embedding meta-pathbased principle into KG embedding task, we formally proposed a novel multiple textual semantic meta-path-based $\mathrm{KG}$ embedding for recommendation technique, called as: MultSemMP-KG2Vec. The proposed MultMP-KG2Vec enables to jointly learn the textual semantic, long-range dependency user-item-specific as well as the multi-typed entities relationships in a given KG. The achieved meta-path-varied representations of users and items are simultaneously fused and transformed in the unified embedding spaces to facilitate the policy-guided sampling process for generating explainable paths over the given $\mathrm{KG}$ in the next step.

- Next, we apply the RL-based approach to tackle the problem of sampling explainable paths for user-item pairs by inherited the soft reward strategy and multi-hop scoring mechanism of previous PGPR [3] model. The path-based reasoning process for generating sets of candidate items is guided by the policy-driven network searching mechanism with the RL learning strategy.

- Finally, we conducted the extensive experiments in standard MovieLens-100K and Amazon datasets, includes: Amazon-Baby, Amazon-Music, Amazon-Book and Amazon-Beauty to evaluate our proposed HINRL4Rec model with recent state-of-the-art explainable personalize recommendation model to demonstrate the effectiveness of our proposed model in this paper.

The rest of our paper is organized into 5 sections. In the next section, we briefly present recent studies related to both traditional, HIN embedding-based and explainable personalized recommendation. In the third section, we formally describe about the explainable recommendation problem, basic concepts and common notations which are used in our paper. In the fourth section, we provided detailed descriptions of our proposed HINRL4Rec model, background ideas and implementations. Next, we demonstrate extensive experiments on benchmark datasets in the fifth section to demonstrate the effectiveness of our proposed HINRL4Rec in comparing with recent recommendation baselines. Finally, in the sixth section, we conclude our works and achievements in this paper as well as highlight some potential directions for the future improvements.

\section{RELATED WORKS \& MOTIVATIONS}

Following different progress milestones of the recommendation domain, the proposed recommendation techniques can be categorized as three main development trends, which are: traditional collaborative filtering, integrated heterogeneous networked data as side information and explainable recommendation.

\subsection{Traditional collaborative filtering approach}

The collaborative filtering (CF) approach is considered as the earliest fundamental baseline for handling recommendation task. The CF-based methods adopt the historical rating data of user-item interactions to learn the latent features of the preferences of user-item relationships for recommendation. Among recommendation-based feature engineering techniques, matrix factorization (MF) is commonly used to analyze and factorize the user-item interaction matrix into low-rank latent feature representations of userspecific and item-specific matrices. Then, these low-rank factorized matrices are utilized to recommend unseen items for specific users. In other words, MF is considered as the primitive feature dimensional reduction approach which are mainly inherited by further advanced techniques in recommendation, such as single value decomposition (SVD), non-negative matrix factorization (NMF), probabilistic matrix factorization (PMF), factorization machine (FM), etc. However, there are major limitations of MF-based methods include the sparsity of user-item interaction as well as the scalability challenges. Therefore, researchers have attempted to associate external auxiliary data with the given user-item interactions to enrich the contextual information of recommendation task. Such as the early proposals of Xie, H. et al. [15] utilized the community information related to user-item interaction to enrich the recommendation scenarios. Or previous words of Liang, D. et al. [16] and Cao, D. et al. [17] in capturing multi-typed latent representation of user-item interactions to improve the accuracy performance of recommendation task. The extended methods have verified the fact that utilizing external auxiliary information can support to effectively leverage the performance of recommendation systems. 


\subsection{Heterogeneous network embedding based approach}

In recent time, heterogeneous network analysis and mining has been widely studied and attracted a significant attention from researchers due to its potential applications in multiple disciplines. A heterogeneous network contains multiple types of nodes/entities and links which fully reflect the real-world datasets like as social networks or knowledge graphs. The meta-path is an important concept of HIN analysis and mining which support to characterize the latent semantic relationships between type-varied nodes/entities. In other word, meta-path is considered as latent features to present the meaningful connectivity between different types of nodes/entities. Early recognizing the powers of HIN in recommendation, $\mathrm{X}$. Yu et al. [18] proposed a novel HIN-based personalized recommendation model by extracting meta-path-based latent features as user-item preferences for leveraging the performance of recommender systems. Similar to that, works of Luo, C. et al. in HETE-CF model [19] and Shi C. et al. in SemRec model [20] proposed the approach of utilizing auxiliary information from heterogeneous like as social networks to improve the performance of personalized recommendation task. These proposed methods have and advantage of flexibly applying meta-path-based feature extraction strategy to capture rich contextual information for recommendation process. However, they are still hindered by mostly relying on the similarity measurement strategies under different semantic meta-paths between user and item nodes/entities. This limitation leads to the incapability of fully capture latent feature representations of typevaried nodes/entities in complex heterogeneous networks, e.g., knowledge graphs or social networks. To challenge this problem, recent advanced representation learning techniques for heterogeneous such as Metapath2Vec [21], Metagraph2Vec [22], PME [23],SHNE [24], etc. have been proposed and demonstrated remarkable outputs for capturing representations of multi-typed nodes/entities and links in a given HIN. By taking advantage of rich-semantic meta-path-based representation learning of multi-typed nodes and relationships in the context of network heterogeneity, the recent HERec [1] model adopted metapath-based random walk mechanism of Metapath2Vec [21] to generate meaningful node/entity sequences which are then used to learn the contextual representation of a given HIN. The different achieved node/entity embedding vectors are then used to facilitate the matrix factorization process for handling recommendation task. HERec has demonstrated significant performances in comparing with recent stateof-the-art recommendation baselines which verified the potential application of HIN-based representation learning in multi-tasked recommender systems. Inherited from the success of HERec model, Zhao, Z. et al proposed the HetNERec [2] model which applies a modified meta-path-based embedding mechanism to fully capture the co-occurring relationships of user-item interactions which enables to better preserve the rich contextual information for recommendation task via extended MF strategy in a given HIN. However, the integrated HIN-based representation learning with MF-based methods encounter a limitation of the difficulty in explaining [25] the achieved recommendation results in the complex latent representations of heterogeneous networks.

\subsection{Path-based explainable recommendation approach}

In recent attempts, Ai Q. et al. proposed a novel integrated knowledge graph based embedding strategy with the path-based fuzzy reasoning over a given $\mathrm{KG}$ to handle the explainable recommendation task. Recent works of Wang, X. et al. proposed a deep recurrent neural network (RNN) based mechanism, called as: KPRN [26] for reasoning over given knowledge graphs for solving the explainable recommendation task. The KPRN iterates all paths between user-item pairs to facilitate the reasoning process for conducting the explainable recommendations. Inspired from path-based reasoning mechanism for recommendation of previous works, Xian, Y. et al. proposed a novel policy-guided reasoning method, called as: PGPR [3] which enables to sample explainable paths of user-item pairs via reinforcement learning strategy for handling personalized recommendation task in large-scale knowledge graphs. Similar to the core principle of PGPR model [3], other path-based reasoning techniques have been adopted this idea to tackle explainable recommendation and achieve significant results in real-world large-scale KGs, like as: SCPR [4] which models conversational recommendation by utilizing a custom interactive path-based reasoning mechanism over KGs or KGPolicy [14] uses the integrated negative sampling technique with reinforcement learning 
based policy network to effectively explore high-quality negative items for training the recommendation model. Even policy-oriented searching mechanism over KGs demonstrate remarkable results in explainable recommendation task, there is still a major limitation which is challenging to implement an effective way to extract useful information from given complex KG's structures in context of heterogeneous networks where semantic paths between nodes/entities in forms of meta-paths have not fully taken in consideration during the $\mathrm{KG}$ embedding process.

\section{Problem formulation \& PRELiminaries}

In this section, we formally formulize the explainable recommendation problem, its related preliminaries as well as background concepts of architectures which are used in our paper.

\subsection{Meta-path-based heterogeneous network representation learning/embedding}

Recently, heterogeneous information network analysis and mining has been becoming a main stream for most of researches related to networked data mining area. The HIN is a special kind of information network which fully reflects real-world networked datasets, like as social networks (Facebook, Twitter, etc.) or knowledge graphs (Wikipedia, YAGO, Freebase, etc.) with large number of type-varied nodes/entities and links/relationships (definition 1). Due to the complexity of real-world heterogeneous networks, the networked data mining paradigm for proposed models must be driven by the meta-level/network's schema related descriptions which support for thoroughly understanding the structures of a given HIN. A knowledge graph (definition 2) is considered as a special type of heterogeneous network which is recently attracted a great deal of attention by researchers due to its potential applications in multiple areas including the knowledge reasoning process for recommendation.

Definition 1: Heterogeneous Information Network (HIN): a HIN is denoted as a graph-based structure: $\mathcal{G}=(\mathcal{V}, \mathcal{E})$, where $\mathcal{V}$ and $\mathcal{E}$ represent for the set of nodes/entities and links/relationships in a given network, respectively. We have two mapping functions, as: $\phi($.$) and \psi($.$) where: \phi: \mathcal{V} \rightarrow \mathcal{A}$ and $\psi: \mathcal{E} \rightarrow \mathcal{R}$ with $\mathcal{A}=\left\{A_{1}, A_{2}, \ldots, A_{|\mathcal{A}|}\right\}$ and $\mathcal{R}=\left\{R_{1}, R_{2}, \ldots, R_{|\mathcal{R}|}\right\}$ are sets of identified nodelentity and link/relationship types, respectively. In homogeneous networks, we have only one type of nodelentity and link/relationship, as: $|\mathcal{A}|=1$ and $|\mathcal{R}|=1$, whereas multiple types of node/entity and link/relationship in a heterogeneous network, as: $|\mathcal{A}| \geq 1$ and $|\mathcal{R}| \geq 1$. A network schema of a given $H I N$ is denoted as: $\mathcal{S}=(\mathcal{A}, \mathcal{R})$.

Definition 2: Knowledge Graph $(\mathbf{K G})$ : is a special type of HIN in form of graph-based structure as: $\mathcal{G}=$ $(\mathcal{V}, \mathcal{E})$ with the sets of type-varied entities $(\mathcal{V})$ and relationships $(\mathcal{E})$. Normally, a knowledge graph is modelled as a set of triples as: $\mathcal{T}=\left\{\left\langle e_{h}, r, e_{t}\right\rangle\right\}$, with: $e_{h}, e_{h} \in \mathcal{V}$ and $e_{t}, e_{t} \in \mathcal{V}$ are the head and tail entities, respectively which are connected by a type-specific relation $r, r \in \mathcal{E}$.

Definition 3: Meta-path (P): is an important concept of heterogeneous network analysis and mining, denoted as a sequential nodelentity path in form of: $A_{1} \stackrel{R_{1}}{\rightarrow} A_{2} \stackrel{R_{2}}{\rightarrow} \ldots \stackrel{R_{l}}{\rightarrow} A_{l+1}$ with (l)-length, a meta-path is also formulized as: $A_{1} A_{2} \ldots A_{l+1}$ for short which represent for a set of composed link/relationship, as: $R=$ $R_{1} \circ R_{2} \circ \ldots \circ R_{l}$, between two nodes/entities belong to $A_{1}$ and $A_{l+1}$ types, respectively.

Definition 4: HIN as Side Information for Recommendation: in the context of rich-schematic recommendation scenario, the user-item pairwise interactions might be associated with other kinds of information and these external informative resources can be modelled as a HIN, as: $\mathcal{G}=(\mathcal{V}, \mathcal{E})$. For recommendation task, we have two main types of nodelentity, which are: user $(u, u \in \mathcal{U})$ and item $(i, i \in$ $\mathcal{J})$, where: $\mathcal{U} \subset \mathcal{V}$ and $\mathcal{J} \subset \mathcal{V}$. For each user-item interaction belongs to a historical rating set $(\mathfrak{N})$ indicates the review/rating relationship between a specific $i^{\text {th }}$ user and $j^{\text {th }}$ item, denoted as a tuple: $\left\langle r_{i, j}, u_{i} i_{j}\right\rangle \in \mathfrak{N}$ or $\mathfrak{N}=\left\{\left\langle r_{i, j}, u_{i} i_{j}\right\rangle\right\}$ with: $\mathfrak{N} \subset \mathcal{E}$.

To model semantic paths between same-typed/different-typed nodes/entities in a given HIN, the meta-paths (definition 3) are used to model latent connectivity features as well as the rich-schematic structure of the overall network. Meta-path is majorly applied in different primitive tasks of HIN analysis and mining such 
as similarity measure, node clustering and recommendation. Recent efforts [18] [19] [20] of utilizing HIN as side information (definition 4) for multi-tasked recommendation demonstrate significant improvements. These works mainly leverage the meta-path-based similarity measurement between user-item pairs and other-typed entities interactions in context of heterogeneity to leverage the performance of recommendation. However, these similarity-based techniques mostly rely on the meta high-dimensional structural analysis and extraction approach to obtain the rich user-item preferences for recommendation and might fail to sufficiently preserve the complex low-dimensional latent features of users and items for recommendation. Therefore, further advances in network embedding area have been adopted to overcome this limitation (definition 5). Recent proposals [1] [2] of taking representations of nodes/entities in heterogeneous networks (e.g., knowledge graphs or social networks) as side information demonstrate significant improvements in recommendation. However, these models still encounter a limitation of unexplainable results for the context of complex reasoning recommendation.

Definition 5: Network Representation Learning (NRL): is mainly inherited from a well-known NLP paradigm, Word2Vec [27] which enables to transform the sets of nodes/entities and links/relationships of a given network, as: $\mathcal{G}=(\mathcal{V}, \mathcal{E})$ - into fixed d-dimensional embedding vectors where still preserves the informative characteristics and proximity structure of given nodes/entities. In general, the ultimate goal of NLR is find a mapping function, denoted as: $f_{N R L}($.$) to learn the embedding representations of nodes, as:$ $f_{N R L}(\mathcal{V}) \rightarrow X_{\mathcal{V}} \in \mathcal{R}^{|\mathcal{V}| \times d}$ or links, as: $f_{N R L}(\mathcal{E}) \rightarrow X_{\mathcal{E}} \in \mathcal{R}^{|\mathcal{E}| \times d}$ in a given network.

\subsection{Explainable recommendation over heterogeneous networks/knowledge graphs}

Definition 6: KG-based Reasoning for Explainable Recommendation: by utilizing a given knowledge graph, denoted as: $\mathcal{G}=(\mathcal{V}, \mathcal{E})$ - with a set of user entities, as: $\mathcal{U} \subset \mathcal{V}$, for each user, as: $u, u \in \mathcal{U}$, we need to sample a set of items, denoted as: $\mathcal{J}_{u}=\left\{\left\langle i_{k}\right\rangle\right\}_{k=1}^{N}$ and $\mathcal{J}_{u} \subset \mathcal{J}$. For each recommended $k^{\text {th }}$ item, as: $\left(i_{k}\right)$ is associated with a specific reasoning path, denoted as: $p\left(u, i_{k}\right)$.

To deal with explainable personalized recommendation task, several path-based reasoning over heterogeneous networks (normally knowledge graphs) methods [3] [4] [14] have been proposed to fully preserve the variety and diversity of user-item interaction over semantic paths for sampling recommended items. This approach can also be called as KG-based reasoning method for explainable recommendation (definition 6). Recent models such as PGPR [4] formulate the recommendation as a reasoning process which stochastically capture the extra relationships among users and items in the real-world networks. Normally, the reinforcement learning is used to formulate and interpret the recommendation reasoning process via different policy-based searching mechanism. However, existing path-oriented reasoning methods still encounter limitations of fully preserving the unified representations of user-item interconnecting relationships via different semantic meta-paths during the knowledge graph embedding process.

To effectively fulfill limitations of both approaches, in this paper we proposed a novel KG embedding based explainable recommendation method which integrates the rich-semantic representations of entities in a given KG into the reinforcement learning based reasoning process for efficiently sampling recommendations for user entities during the inference phase. Table 1 lists common notations which are used in our paper and their detailed descriptions.

Table 1. List of notations and descriptions in this paper

\begin{tabular}{|c|l|}
\hline Notation & \multicolumn{1}{|c|}{ Description } \\
\hline $\mathcal{G}=(\mathcal{V}, \mathcal{E})$ & $\begin{array}{l}\text { A homogeneous/heterogeneous network as a graph-based structure } \\
\text { with } \mathcal{V} \text { and } \mathcal{E} \text { the sets of nodes/entities and links/relationships, } \\
\text { respectively. }\end{array}$ \\
\hline $\mathcal{S}=(\mathcal{A}, \mathcal{R})$ & $\begin{array}{l}\text { A network schema of a heterogeneous information network with } \mathcal{A} \text { and } \\
\mathcal{R} \text { are the sets of node/entity and link/relationship types. }\end{array}$ \\
\hline $\mathcal{U}$ and $\mathcal{J}$ & The sets of user and item/product entities, respectively. \\
\hline $\mathfrak{P}$ and $\mathcal{P}$ & A set of meta-paths and a meta-path, respectively. \\
\hline
\end{tabular}




\begin{tabular}{|c|l|}
\hline$p$ & $\begin{array}{l}\text { A recommendation-aware reasoning path in forms of an unsymmetric } \\
\text { meta-path. }\end{array}$ \\
\hline$w, s$ and $d$ & A word, sentence and a document, respectively. \\
\hline$\vec{e}$ & A general embedding vector. \\
\hline $\overrightarrow{e_{M P}}$ & A meta-path-based embedding vector. \\
\hline $\overrightarrow{e_{K G}}$ & A translation-based KG embedding vector. \\
\hline $\overrightarrow{e_{B E R T}}$ & A BERT-based embedding vector. \\
\hline $\overrightarrow{e_{B L-L S T M}}$ & A Bi-LSTM based embedding vector. \\
\hline $\mathfrak{S}$ & A state of a given agent. \\
\hline $\mathcal{A}$ & A set of action space for a given agent. \\
\hline $\mathfrak{r}_{\mathfrak{I}}$ & A soft reward for an agent when it reaches the terminal state $(\mathfrak{I})$. \\
\hline$\Pi($.$) and \Phi()$. & The policy network and value network of a given model. \\
\hline
\end{tabular}

\section{Methodology \& IMPLEMENTATIONS}

In this section, we formally present the methodology and detailed implementations of our proposed HINRL4Rec model. In our proposed HINRL4Rec model, we first apply a custom meta-path-based embedding technique called as MultMP-KG2Vec to effectively learn the rich-semantic representations of user and item entities in a given knowledge graph as a heterogeneous network. Then, we apply a personalized non-linear embedding fusion mechanism to merge meta-path-varied embedding vectors of user and item entities into unified embedding spaces which are then jointly optimized with the KG embedding process via the TransE model [6]. Next, we apply the reinforcement learning approach with policy-guided reasoning procedure to sample possible explainable item-driven paths for each user in order to conduct the recommendation.

\subsection{MultSemMP-KG2Vec: rich-schematic KG embedding approach for recommendation}

First of all, in order to stimulate the product/item recommendation for a list of users in a given heterogeneous network by utilizing the knowledge graphs as side information, we apply the meta-pathbased embedding mechanism of Metapath2Vec [23] model to capture rich-semantic path-based representation of user and item entities in a given network.

\subsubsection{Recommendation-driven meta-path-based $K G$ embedding approach}

In a given KG as a heterogeneous network, we apply the meta-path-based random walk mechanism to generate rich-semantic and meaningful node/entity sequences for each user and item node. In fact, in a complex and rich-schematic HIN, each user-user and item-item pair might be connected by multiple interconnecting relationships in forms of meta-paths, e.g., for user entity: $U[$ user $] \stackrel{\text { friend }}{\longrightarrow} U(U-U), U$ $\stackrel{\text { review }}{\longrightarrow} \mathrm{P}[$ product $] \stackrel{\text { review }}{\longleftarrow} \mathrm{U}(\mathrm{U}-\mathrm{P}-\mathrm{U})$, etc. and for item/product entity: $\mathrm{P} \stackrel{\text { reviewed-by }}{\longrightarrow} \mathrm{U} \stackrel{\text { reviewed-by }}{\longleftarrow} \mathrm{P}(\mathrm{P}-\mathrm{U}-\mathrm{P})$, $\mathrm{P} \stackrel{\text { belong }}{\longrightarrow} \mathrm{C}[$ category $] \stackrel{\text { belong }}{\longleftarrow} \mathrm{P}(\mathrm{P}-\mathrm{C}-\mathrm{P})$, etc. To formulate the meta-path-varied embedding process over a given HIN for recommendation, we formulate the sets of related meta-paths for user and item entities, as: $\mathfrak{P}^{\mathcal{U}}=\left\{\mathcal{P}_{1}^{u}, \mathcal{P}_{2}^{u}, \ldots, \mathcal{P}_{\left|\mathfrak{P}^{u}\right|}^{u}\right\}=\left\{\mathcal{P}_{i}^{u}\right\}_{i}^{\left|\mathfrak{P}^{u}\right|}$ and $\mathfrak{P}^{\mathcal{J}}=\left\{\mathcal{P}_{1}^{\mathcal{J}}, \mathcal{P}_{2}^{\mathcal{J}}, \ldots, \mathcal{P}_{\left|\mathfrak{P}^{\mathcal{J}}\right|}^{\mathcal{J}}\right\}=\left\{\mathcal{P}_{i}^{\mathcal{J}}\right\}_{i}^{\left|\mathfrak{P}^{\mathcal{J}}\right|}$, respectively. For each meta-path $(\mathcal{P})$ which links two pairwise user-user or item-item entities, the random walks are generated over a given heterogenous network, as: $\mathcal{G}=(\mathcal{V}, \mathcal{E})$, following the probabilistic distribution of starting at a specific entity $(x)$ and moving to a next entity $(v)$, denoted as: $\pi_{\mathrm{x} \sim \mathrm{v}}, \mathcal{P}$ (as shown in equation 1 ):

$$
\pi_{\mathrm{x} \sim \mathrm{v}}, \mathcal{P}=\left\{\begin{array}{c}
\text { with: } \mathrm{e}(\mathrm{x}, \mathrm{v}) \in \mathcal{E}\left\{\begin{array}{c}
\phi(\mathrm{v})=\mathrm{t}+1, \frac{1}{|\operatorname{Neighbor}(\mathrm{x})|} \\
\phi(\mathrm{v}) \neq \mathrm{t}+1,0
\end{array}\right. \\
\text { with: } \mathrm{e}(\mathrm{x}, \mathrm{v}) \in \mathcal{E} \text { and } \phi(\mathrm{x}) \neq \phi(\mathrm{v}), 0 \\
\text { with: } \mathrm{e}(\mathrm{x}, \mathrm{v}) \notin \mathcal{E}, 0
\end{array}\right.
$$


Where:

- $\mathrm{e}(\mathrm{x}, \mathrm{v})$ and Neighbor(x), are and directed edge between two $(x)$ and $(v)$ entities and $\mathrm{e}(\mathrm{x}, \mathrm{v}) \in \mathcal{E}$ and a set of neighborhood entities of the given $(x)$ entity.

- $\quad[t+1]$, is the expected next type of a given (v) entity following the defined meta-path: $\mathcal{P}$.

From the generated node sequences via meta-path-based random walk, we apply the Skip-gram architecture with a single-hidden layer neural network to learn the representations of user and item entities as the homogeneous representation learning approach by truncating non-user/item-typed entities in the target sampled walks. Since our ultimate purpose in this paper is taking the rich-semantic representations of multityped entities in HIN to improve the performance of recommendation which is tightly associated with the user and item entities (known as: target-typed entities). Therefore, we filter all non-target-typed entities, such as: categories, brands, etc. from the generated meta-path-based walks in previous step which make our embedding approach is considered as the homogeneous-driven representation learning strategy. Hence, our proposed model learns the representations of user/item entities by using homogeneous neighborhood. In fact, the set of sampled type-varied entities which are constructed by using meta-path-based random walk is considered rich semantic due to the random walkers have been travelled through all types of entities following the defined meta-path. Therefore, even we apply non-target-typed entity filtering the rich semantic relationships between type-varied entities still be sufficiently captured and preserved in a heterogeneous contextual embedding space. By applying this filtering mechanism, it not only supports to optimize the recommendation-aware learning purpose but also speed-up the model training process. Moreover, by using non-target-typed entity filtering, we also can transform user/item entities in the same embedding space which relaxes the sparsity and non-relatedness challenge of representing all the heterogeneous entities in a unified network embedding space. Similar to previous homogeneous network representation learning models [8] [10], we defined a learning objective of maximizing the appearance probability of contextual/neighborhood entities, denoted as: $\left(\mathrm{C}_{\mathrm{e}}\right)$, which are generated and filtered by the meta-path-based random walk strategy, for each target-typed entity, denote as: (e), as the following (as shown in equation 2):

$$
\max _{\mathrm{f}_{\mathrm{NRL}}} \sum_{\mathrm{e} \in \mathcal{V}} \log \operatorname{Prob}\left(\mathrm{C}_{\mathrm{e}} \mid \mathrm{f}_{\mathrm{NRL}}(\mathrm{e})\right)
$$

Where:

- $\mathrm{C}_{\mathrm{e}}$, is a set of contextual/neighborhood entities of the given target-typed entity: (e).

- $\mathrm{f}_{\mathrm{NRL}}($.$) , is the defined network embedding function which is used to transform a given entity (e)$ into the fixed d-dimensional embedding vector or $\mathrm{f}_{\mathrm{NRL}}(\mathrm{e}) \in \mathbb{R}^{1 \times d}$.

In order to learn the parameters of the given $\mathrm{f}_{\mathrm{NRL}}($.$) function, we apply the Skip-gram architecture [27]$ with stochastic gradient descent (SGD) strategy. Specifically, in the context of user/item-contextual entities optimization scenario, to achieve the likelihood conditional probability of having a set of generated contextual entities $\left(C_{e}\right.$ for a specific entity $(e)$, as the: $\operatorname{Prob}\left(C_{e} \mid f_{N R L}(e)\right)$ is normally calculated as a softmax function, denoted as: $\operatorname{Prob}\left(\mathrm{C}_{\mathrm{e}} \mid \mathrm{f}_{\mathrm{NRL}}(\mathrm{e})\right)=\frac{\exp \left(\mathrm{X}_{\mathrm{e}}^{t} \cdot \mathrm{X}_{\mathrm{e}}^{t}\right)}{\sum_{\mathrm{v} \in \mathcal{V}^{t}} \exp \left(\mathrm{X}_{\mathrm{v}}^{t} \mathrm{X}_{\mathrm{e}}^{t}\right)}$, where: $\mathrm{X}^{t} \in \mathbb{R}^{\mathcal{V}^{t} \times \mathrm{d}}$ is the achieved embedding matrix of a given $t^{t h}$ target-typed entities (user/item). To achieve the representation embedding matrix of $\mathrm{X}^{t}$, we defined a training objective function with negative sampling method as: $\mathcal{O}\left(\mathrm{X}^{t}\right)=$ $\log \sigma\left(\mathrm{X}_{\mathrm{C}_{\mathrm{e}}^{t}}^{t} \cdot \mathrm{X}_{\mathrm{e}}^{t}\right)+\sum_{h=1}^{\text {neg-size }} \mathbb{E}_{\mathrm{v}^{h} \sim \mathbb{P}(\mathrm{v})}\left[\log \sigma\left(-\mathrm{X}_{\mathrm{v}^{h}}^{t} \cdot \mathrm{X}_{\mathrm{e}}^{t}\right)\right]$, with $\sigma($.$) and \mathbb{P}(\mathrm{v})$ are the sigmoid function and the set of sampled entities by probabilistic drawing $\mathrm{v}^{k}$ negative entity with neg-size times, respectively. Then, we updated parameters of the defined network representation function, as: $\mathrm{f}_{\mathrm{NRL}}($.$) with the calculated$ gradients as: $\mathrm{X}^{t}=\mathrm{X}^{t}-\eta \frac{\partial \mathcal{O}(\mathrm{X})}{\partial \mathrm{X}_{e}}$ by using SGD with $(\eta)$ is the predefined learning rate. Then, for a set of meta-paths of $t^{t h}$ target type, as: $\mathfrak{P}^{t}$, we achieve a set of meta-path-varied embedding matrices, denoted 
as: $\mathbb{E}^{t}=\left\{\mathrm{X}^{t, \mathcal{P}}\right\}_{\mathcal{P} \in \mathfrak{P}^{t}}$ and for a specific (i ${ }^{\text {th }}$ ) entity belongs to $(t)$ target type, we have a set of embedding vector as: $\mathrm{E}_{\mathrm{i}}^{t}=\left\{\overrightarrow{\mathrm{e}_{1}^{t, \mathcal{P}}}\right\}_{\mathcal{P} \in \mathfrak{P}^{t}}$, with each meta-path-based embedding vector: $\overrightarrow{\mathrm{e}^{t, \mathcal{P}}} \in \mathrm{X}^{t, \mathcal{P}}$. To conveniently transform these meta-path-varied embedding vectors of each $\left(\mathrm{i}^{\text {th }}\right)$ entity into a single unified embedding space for further usage in the next KG embedding steps, we apply a simple linear fusion function, denoted as: fuse $\mathrm{I}_{\mathrm{l}}($.$) in order to map these meta-path-varied embedding vectors \left(\mathrm{E}_{\mathrm{i}}^{t}\right)$ of a specific $\left(\mathrm{i}^{\text {th }}\right)$ entity, into a unified meta-path-based embedding vector, denoted as: $\left(\overrightarrow{\mathrm{e}_{\mathrm{MP}}^{t}}\right)$, by applying a linear merging layer as the following (as shown in equation 3):

$$
\begin{aligned}
\overrightarrow{\mathrm{ePP}_{1}^{t}}=\text { fuse }_{1}\left(\mathrm{E}_{\mathrm{i}}^{t}\right. & \left.=\left\{\overrightarrow{\mathrm{e}_{1}^{t, \vec{P}}}\right\}_{\mathcal{P} \in \mathfrak{P}^{t}}\right)=\frac{1}{\left|\mathfrak{P}^{t}\right|} \sum_{\mathcal{P} \in \mathfrak{P}^{t}}\left(\mathrm{U}^{\text {fuse }_{1}} \cdot \overrightarrow{\mathrm{e}_{1}^{t, \mathcal{P}}}+\mathrm{b}_{\alpha}^{\text {fuse }} \mathrm{e}_{1}\right) \\
\mathrm{U}^{\text {fuse }_{1}} & =\operatorname{Dropout}\left(\operatorname{ReLU}\left(\mathrm{W}^{\text {fuse }_{1}}+\mathrm{b}_{\beta}^{\text {fuse }_{1}}\right)\right)
\end{aligned}
$$

Where:

- $\overrightarrow{\mathrm{e}_{\mathrm{MP}} t}$, meta-path-based fused embedding of $\left(i^{t h}\right)$ entity belongs to a specific $(t)$ target type.

- $\quad \mathrm{U}^{\text {fuse }_{1}, \mathrm{~W}^{\text {fuse }_{1}}, \mathrm{~b}_{\alpha}^{\text {fuse }} \mathrm{e}_{1}}$ and $\mathrm{b}_{\beta}^{\text {fuse }} \mathrm{e}_{1}$ are model's weighting and bias parameter matrices of a given linear fusion function which will be updated during the model training process.

By using a linear fusion layer which is similar to the multi-layered perceptron architecture, we can transform all different meta-path-based embeddings of each target-typed entity into a unified target representation space and still sufficiently preserving the semantic relationships between entities in a given KG. Then, the achieved unified embedding vectors of these target-typed entities are used to facilitate the KG embedding process in the next step.

\subsubsection{Integrated textual semantic representation with target-typed entities}

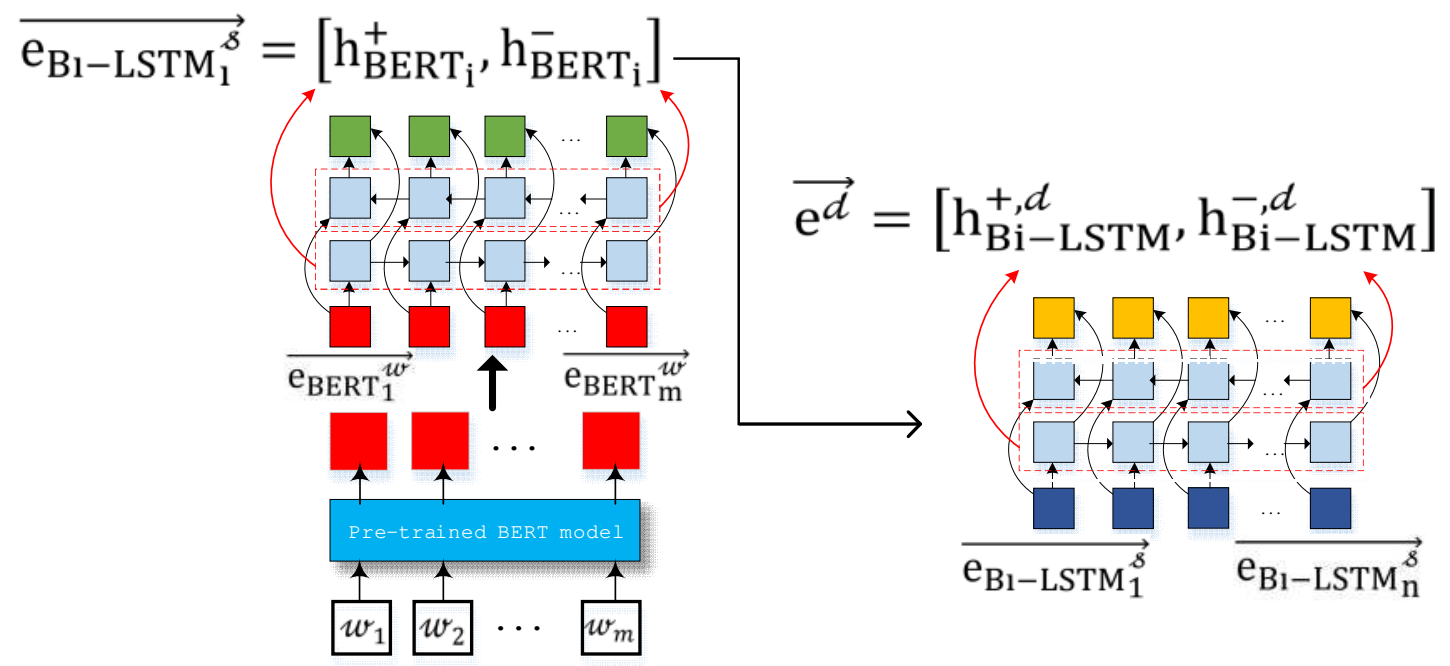

Figure 2. The illustrations of overall textual embedding process for associated textual data of target-typed entities in a given knowledge graph via BERT and Bi-LSTM encoder

For the further extensions on rich textual semantic integration with the learnt representations of target-typed entities (e.g., products/items, etc.) in the recommendation task, we firstly apply the BERT-based textual encoder to learn the representations of sentences in given textual data of target-typed entities. Then, we use a Bi-LSTM encoder [28] to learn the full-textual sequential representations of the given textual data. Finally, the achieved textual embedding vectors are concatenated into its targeted entity in order to enrich the latent feature representation of a given entity in both meta-path-based structural and textual semantic 
meanings. To do this, giving a descriptive data of a target-typed entity (e.g., product's descriptions, overviews/short descriptions of movies, etc.) as a textual document with (n) number of sentences, as: $d=$ $\left\{s_{1}, s_{2}, \ldots, s_{\mathrm{n}}\right\}$, we firstly use BERT to learn the sequential representations of (m) number of words in each

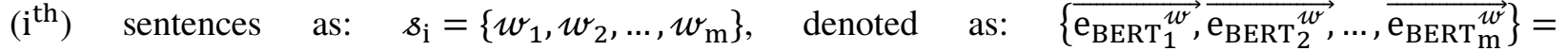
$\operatorname{BERT}\left(\left\{w_{1}, w_{2}, \ldots, w_{m}\right\}\right)$. Then, to learn the completed embedding of a given sentence $s_{\mathrm{i}}$, we use a BiLSTM encoder to effectively aggregate and capture the sequential information of occurring words in the given sentence, denoted as: $\overrightarrow{\mathrm{e}_{\mathrm{B} 1-\mathrm{LSTM}_{1}^{8}}}$. Our sequential textual embedding technique for each sentence in this case can be formulated as shown in the equation 4 (as illustrated in Figure 2-A):

$$
\begin{aligned}
& \mathrm{h}_{\mathrm{BERT}_{\mathrm{i}}}^{+, s}=\operatorname{LSTM}_{s}^{+}\left(\left\{\overrightarrow{\mathrm{e}_{\mathrm{BERT}_{j}}{ }_{j}}\right\}_{\mathrm{j}=1}^{\mathrm{m}}, \theta_{s}^{+}\right) \\
& \mathrm{h}_{\mathrm{BERT}_{\mathrm{i}}}^{-, s}=\operatorname{LSTM}_{s}^{-}\left(\left\{\overrightarrow{\mathrm{e}_{\text {BERT }}{ }_{\mathrm{j}}},\right\}_{\mathrm{j}=1}^{\mathrm{m}}, \theta_{s}^{-}\right) \\
& \overrightarrow{\mathrm{e}_{\mathrm{B} 1-\mathrm{LSTM}_{1}^{8}}}=\left[\mathrm{h}_{\mathrm{BERT}_{\mathrm{i}}}^{+}, \mathrm{h}_{\mathrm{BERT}}^{-}\right] \\
& \mathrm{h}_{\mathrm{Bi}-\mathrm{LSTM}}^{+, d}=\operatorname{LSTM}_{d}^{+}\left(\left\{\overrightarrow{\mathrm{e}_{\mathrm{B} 1-\mathrm{LSTM}_{1}}}\right\}_{\mathrm{i}=1}^{\mathrm{n}}, \theta_{d}^{+}\right) \\
& \mathrm{h}_{\mathrm{Bi}-\mathrm{LSTM}}^{-, d}=\operatorname{LSTM}_{d}^{-}\left(\left\{\overrightarrow{\mathrm{e}_{\mathrm{B} 1-\mathrm{LSTM}_{1}}}\right\}_{\mathrm{i}=1}^{\mathrm{n}}, \theta_{d}^{-}\right) \\
& \overrightarrow{\mathrm{e}^{d}}=\left[\mathrm{h}_{\mathrm{Bi}-\mathrm{LSTM}}^{+, d}, \mathrm{~h}_{\mathrm{Bi}-\mathrm{LSTM}}^{-, d}\right]
\end{aligned}
$$

Where:

- $\left[\theta^{+}, \theta^{-}\right]$, are the model's parameters of a given Bi-LSTM architecture for both two forward and backward directions, respectively.

- $\left[\mathrm{h}^{+}, \mathrm{h}^{-}\right]$, presents for the concatenation of two directional hidden states of a given Bi-LSTM architecture to produce a final embedding vector.

Then, after achieving the textual representation of each sentence in a given document (d), we also apply another Bi-LSTM encoder to learn the sequential latent features of all sentences and aggregated into a unified space by concatenating the output hidden states (as shown in the equation 5) in order to achieve the final embedding of each document $(d)$, denoted as: $\overrightarrow{\mathrm{e}^{d}}$ (as illustrated in Figure 2-B). The outputs of this textual embedding process will be a set of target-typed textual embedding vectors. These textual embedding vectors are then merged into the previous achieved $(t)$-typed meta-path-based embedding vectors via max pooling strategy, denoted as: $\operatorname{MaxPool}\left(\overrightarrow{\mathrm{e}_{\mathrm{MP}_{1}^{t}}}, \overrightarrow{\mathrm{e}_{1}^{t, d}}\right)$ to significantly encapsulate both textual and structural latent representations of target-typed entities in the context of rich content-based heterogeneous network.

\subsubsection{Recommendation-aware knowledge graph embedding with joint optimization of meta-path- based representation learning}

Furthermore, to learn directed relationships between entities in a given KG, we simply apply the translation mechanism of TransE model to learn the ad-hoc representations of the KG's relationships in forms of triples, denoted as: $\left\langle e_{h}, r, e_{t}\right\rangle$, with $e_{h}$ and $e_{t}$ are the head and tail entities which are connected by a specific relation (r). In the TransE model, the training objective is implicit as the embedding vector of tail entity, as: $\overrightarrow{\mathrm{e}_{\mathrm{t}}}$ should be close to the embedding vector of head entity, as: $\left(\overrightarrow{\mathrm{e}_{\mathrm{h}}}\right)$ plus existed relationship embedding vectors, as: $\vec{r}$. This KG representation learning purpose is generally formulated as: $\overrightarrow{e_{h}}+\vec{r}=\overrightarrow{e_{t}}$. To effectively learn the embedding vectors of entities and relations in a given $\mathrm{KG}$, the margin-based ranking minimization process [5] is applied with the defined loss function for the training process, as: $\mathcal{L}=$ $\sum_{\left(\mathrm{e}_{\mathrm{h}}, \mathrm{r}, \mathrm{e}_{\mathrm{t}}\right) \in \mathcal{T}} \sum_{\left(\mathrm{e}_{\mathrm{h}}^{\prime}, \mathrm{r}, \mathrm{e}_{\mathrm{t}}^{\prime}\right) \in \mathcal{T}^{\prime}}\left(\gamma+\mathrm{d}\left(\overrightarrow{\mathrm{e}_{\mathrm{h}}}+\overrightarrow{\mathrm{r}}, \overrightarrow{\mathrm{e}_{\mathrm{t}}}\right)-\mathrm{d}\left(\overrightarrow{\mathrm{e}_{\mathrm{h}}^{\prime}}+\overrightarrow{\mathrm{r}}, \overrightarrow{\mathrm{e}_{\mathrm{t}}^{\prime}}\right)\right)_{+}$with $\gamma$ and $\mathrm{d}($.$) is the margin hyper-$ parameter and squared Euclidean distance function. However, the TransE model only supports to learn the 
ad-hoc relationships of entities in a given $\mathrm{KG}$ instead of the rich semantic representations of target-typed entities (e.g., users and products/items) via different inter-connecting relationships. Therefore, we need to inject the achieved rich-semantic meta-path-based representations of target-typed entities in previous steps into the representation learning process of target-typed entities for sufficiently preserving the complex structure of a given KG in the context of network heterogeneity. To do this, we softly fuse the meta-pathbased embedding vectors into the TransE-based embedding vector of same target-typed entities during the KG embedding process by using a non-linear personalized fusion function, denoted as: fuse $\mathrm{pnl}_{(}($.$) . Let$ simply denote an (i $\left.{ }^{\text {th }}\right)$ TransE-based embedding vector of the $(t)$ target-typed entity as: $\overrightarrow{\mathrm{e}_{\mathrm{KG}_{1}}}$ we apply a non-linear fusion layer to merge the target-typed entity: $\overrightarrow{\mathrm{eG}_{1}}$ into its associated unified meta-path-based embedding vector in previous steps, denoted as: $\overrightarrow{\mathrm{eP}_{1}}$, to produce a final merged embedding vector, denoted as: $\overrightarrow{e_{l}}$. The personalized non-linear layer is used in our case is formally defined as the following (as shown in equation 6):

$$
\begin{aligned}
& \overrightarrow{\mathrm{e}_{1}}=\text { fuse }_{\mathrm{pnl}}\left(\overrightarrow{\mathrm{e}_{\mathrm{KG}_{1}}}, \overrightarrow{\mathrm{e}_{\mathrm{MP}_{1}}}\right)=\sigma\left(\mathrm{U}^{\text {fuse }}{ }_{\mathrm{pnl}} \cdot \operatorname{MaxPool}\left(\overrightarrow{\mathrm{e}_{\mathrm{KG}_{1}}}, \overrightarrow{\mathrm{e}_{\mathrm{MP}_{1}}}\right)+\mathrm{b}_{\alpha}^{\text {fuse }_{\mathrm{pnl}}}\right) \\
& \mathrm{U}^{\text {fuse }_{\mathrm{pnl}}}=\operatorname{Dropout}\left(\sigma\left(\mathrm{W}^{\text {fuse }_{\mathrm{pnl}}}+\mathrm{b}_{\beta}^{\text {fuse }_{\mathrm{pnl}}}\right)\right)
\end{aligned}
$$

Where:

- $\sigma($.$) , is the sigmoid function, denoted as: \sigma()=.\frac{1}{1+e^{-}}$.

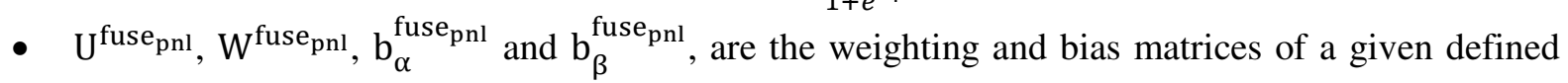
personalized non-linear. These parameters will be updated during with the TransE-based knowledge graph process.

To flexibly join the complex and rich semantic structures of meta-path-based and translation-based (via TransE model) embedding vectors of each target-typed entities in a given KG, we apply non-linear fusion layer with a sigmoid function to enable the capability of our model for deeply learning latent characteristic features as well as effectively transforming them into the target embedding space for facilitating the recommendation task. Our practical implementations have showed that the sigmoid-based linear fusion mechanism achieved better performance in learning and merging the latent features of both meta-pathbased and translation-based embedding vectors of KG's entities than using the full-connected linear one. Similar to the linear fusion layer which is used to combine meta-path-based embedding vectors of KG's entities in previous steps, the parameters of fuse $\mathrm{pnl}_{\mathrm{p}}($.$) function are also simultaneously updated during the$ KG embedding process. The final unified representations of these target-typed entities are then used to facilitate the policy-oriented path reasoning process as the Markov Decision Process (MDP) [29] of reinforcement learning area for handling explainable recommendation task.

\subsection{Explainable recommendation over KG by using reinforcement learning}

There are advantages for stimulating the recommendation tasks as a reinforcement-learning-based path searching problem with the policy-guided reasoning process. First of all, in such large-scale KGs/social networks (e.g., Facebook, Amazon, Douban, etc.) with huge number of entities and relationships, fetching all possible paths which link user-item pairs is considered as a high-pressured and time-consuming task as well as ineffective for practical implementation with the rapid evolution of real-world KGs/social networks. Therefore, it might be challenging to perform an effective user-item pairwise relationship pruning and finding relatedness paths between user-item pairs as a heuristic procedure. Majorly inspired from previous works of Xian, Y. et al. in the PGPR [3], we take the advantages of the policy-guided path finding mechanism to generate sequential entities between user-item pairs as meaningful paths which help to extract explainable candidate items of each user within a given KG. The core principle of our approach is formulated the recommendation as a MDP problem where our model's reasoning mechanism/agent tries to make graph-based transitions over a given KG as the environment to identify the potential items which are 
qualified by the starting user entities. To do this, the recommendation task is mathematically formulated with the intuitions of the again-environment relationships, the action spaces, state at each timestep and the rewarding mechanism.

First of all, we need to define the state for each graph-based transition over a given KG. As the recommendation-driven approach, for each state at a specific $\left(\mathrm{t}^{\text {th }}\right)$ timestep, denoted as: $\mathfrak{s}_{\mathfrak{t}}$ is formally defined as a tuple, as: $\mathfrak{s}_{\mathfrak{t}}=\left\langle\mathrm{u}, \mathrm{e}^{\mathrm{t}}, \mathfrak{h}^{\mathrm{t}}\right\rangle$, where $\mathrm{u}, \mathrm{e}^{\mathrm{t}}$ and $\mathfrak{h}^{\mathrm{t}}$ are the sets of starting user entity, an entity which the agent has moved to at a specific $\left(\mathrm{t}^{\mathrm{th}}\right)$ timestep and $(k)$-range historical data of current state, respectively. For the (k)-range historical data of each state is an aggregated collection of all travelled entities and their associated relationships from the past $\left(k^{\text {th }}\right)$ steps to the current $\left(\mathrm{t}^{\text {th }}\right)$ timestep, denoted as: $\mathfrak{h}^{\mathrm{t}}=$ $\left\{\mathrm{e}^{\mathrm{t}-k}, \mathrm{r}^{\mathrm{t}-(k+1)}, \ldots, \mathrm{e}^{\mathrm{t}-1}, \mathrm{r}^{\mathrm{t}}\right\}$ with: $\mathrm{t}>k$. Since, the path-based sampling process in a given $\mathrm{KG}$ is considered as endless procedure, hence we need to define a fix terminal threshold for each episode, as: $\mathfrak{I}$, and the end/terminal state for each state is denoted as: $\mathfrak{s}_{\mathfrak{I}}=\left\langle\mathrm{u}, \mathrm{e}^{\mathfrak{T}}, \mathfrak{h}^{\mathfrak{T}}\right\rangle$. For the action space of our agent at a specific $\left(\mathrm{t}^{\text {th }}\right)$ timestep, denoted as: $\mathcal{A}_{t}$ is defined as all possible outgoing links of the current staying entity $\left(\mathrm{e}^{\mathrm{t}}\right)$ at the specific $\left(\mathrm{t}^{\mathrm{th}}\right)$ timestep except the travelled entities which are recorded until the current $\left(\mathrm{t}^{\mathrm{th}}\right)$ timestep, denoted as: $\mathcal{H}$, which is simply a set of already-visited entities by our agent, as: $\mathcal{H}=\left\{\mathrm{e}_{\mathrm{i}}\right\}_{\mathrm{i}=0}^{\mathrm{t}}$. Generally, this possibility of choosing a next entity starting current entity $\left(\mathrm{e}^{\mathrm{t}}\right)$ via different links can be formulated as a random work process with the probabilistic distribution equal to: $\frac{1}{\mid \text { Neighbor }\left(\mathrm{e}^{\mathrm{t}}\right)-\mathcal{H} \mid}$.

In more details, the action space of our agent at the specific $\left(\mathrm{t}^{\mathrm{th}}\right)$ timestep can be formulated as: $\mathcal{A}_{\mathrm{t}}=$ $\left\{\left(\left(\mathrm{r}_{\text {next }}, \mathrm{e}_{\text {next }}\right)\right.\right.$, with: $\forall \mathrm{r}_{\text {next }} \in \mathcal{E}$ and $\forall \mathrm{e}_{\text {next }} \in \mathcal{V}$ and $\forall \mathrm{e}_{\text {next }} \notin \mathcal{H} \mid \mathrm{t}:\left(\mathrm{e}^{\mathrm{t}}, \mathrm{r}, \mathrm{e}\right)$, with: $\left.\left.\forall \mathrm{t} \in \mathcal{T}\right)\right\}$ within a given knowledge graph, as: $\mathcal{G}=(\mathcal{V}, \mathcal{E})$, which is normally composed as a set of triples $(\mathcal{T})$. After identifying the state and action space for our agent, we need to specify the reward for our agent in each step, because in the context of knowledge graph traversal, we have no predefined should-be-achieved targets for our itemoriented (e.g., set of items which are considered as potential for each user) path-based searching process. Therefore, we apply the same recommendation-aware soft rewarding scenario of PGPR [3] which forces the agent should find as much as possible paths which link the target user (u) and their "might-beinteresting" items and at the end/terminal state $\left(\mathfrak{s}_{\mathfrak{T}}\right)$, we reward our agent with a soft recommendationaware terminal reward score, denoted as: $\mathfrak{r}_{\mathfrak{T}}\left(\mathrm{u} \mid \mathrm{e}^{\mathfrak{T}}\right)$ which is calculated by the following (as shown in equation 7):

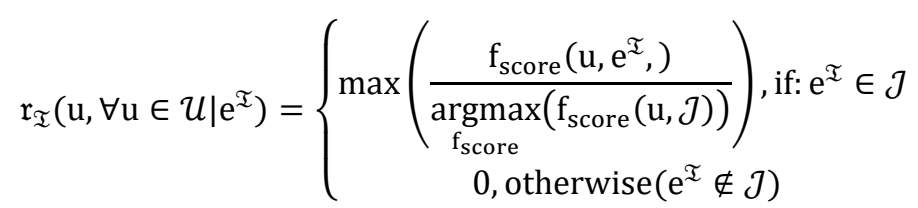

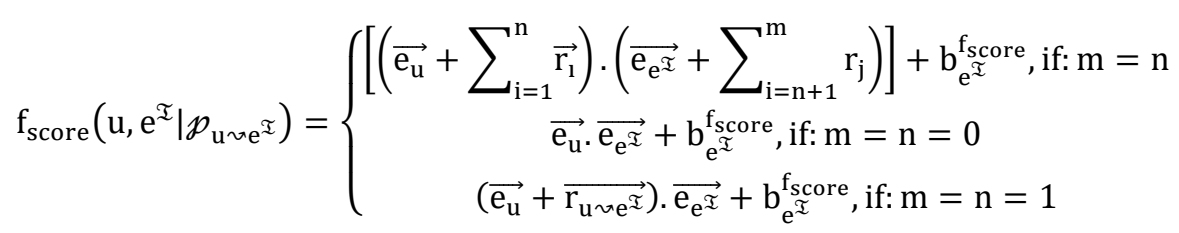

Where:

- $\quad \overrightarrow{\mathrm{e}}$ and $\vec{r}$, are the fused meta-path-based and translation-based embedding vectors for a specific entity which are achieved in previous steps and translation-based embedding vector of a specific relation by using TransE model, respectively.

- $\quad p_{\mathrm{u} \sim \mathrm{e}^{\mathfrak{T}}}$, presents for a $(\mathrm{m})$-length multi-hop-based path between a specific user $(\mathrm{u})$ and a given reached entity $\left(\mathrm{e}^{\mathfrak{I}}\right)$ at the terminal state $(\mathfrak{I})$. In this reward mechanism, we reapply the ideas of 1 reserve multi-hop pattern of PGPR [3] for evaluating and measuring the semantic distance between target-typed user-item via their inter-connecting relationships in forms of multi-hop-based paths. 
In 1-reserve multi-hop pattern evaluation, a given path is symmetrically composed as the backward and forward directions which are divided at the (n)-hop, as: $p_{u \sim e^{\mathfrak{T}}}=u \stackrel{\mathrm{r}_{1}}{\rightarrow} \ldots \stackrel{\mathrm{r}_{n}}{\rightarrow}\left[\mathrm{e}_{\mathrm{n}}\right] \stackrel{\mathrm{r}_{\mathrm{n}+1}}{\longleftarrow} \ldots \mathrm{e}^{\mathfrak{T}}$.

The defined scoring mechanism will return a normalized value, as: $\mathfrak{r}_{\mathfrak{I}}\left(u \mid e^{\mathfrak{T}}\right) \in[0,1]$, for each terminal state of a specific user $(u)$. As the graph-based transitional process, a specific state for each step is explicitly determined by position of the current entity $\left(\mathrm{e}^{\mathrm{t}}\right)$, hence the probabilistic transition from the current $\left(\mathrm{t}^{\mathrm{th}}\right)$ state $\left(\mathfrak{s}_{\mathrm{t}}\right)$ with a specific action, as: $\mathrm{a}_{\mathrm{t}}, \mathrm{a}_{\mathrm{t}} \in \mathcal{A}_{\mathrm{t}}$, to the next $(\mathrm{t}+1)^{\text {th }}$ state $\left(\mathfrak{s}_{\mathrm{t}+1}\right)$ is denoted as: $\operatorname{Prob}\left[\mathfrak{s}_{t+1} \mid \mathfrak{s}_{\mathfrak{t}}, a_{t}\right]=\operatorname{Prob}\left[\left\langle\mathrm{u}, \mathrm{e}^{\mathrm{t}+1}, \mathfrak{h}^{\mathrm{t}+1}\right\rangle \mid\left\langle\mathrm{u}, \mathrm{e}^{\mathrm{t}}, \mathfrak{h}^{\mathfrak{t}}\right\rangle,\left(\mathrm{r}^{\mathrm{t}+1}, \mathrm{e}^{\mathrm{t}+1}\right)\right]=1$. For simple implement, our proposed path-based reasoning process assumes the prior distribution of all user entities as the uniform distributions, so all the user entities have an equal probability to be picked up at the beginning step. Finally, we apply the training by using the policy network, denoted as: $\Pi(. \mid \overrightarrow{\mathfrak{s}}, \mathcal{A}(\mathrm{u}))$ and the value network, denoted as: $\Phi(\overrightarrow{\mathfrak{s}})$ and these two networks have the same hidden layers. The structures of these two networks are formulated as the following (as shown in equation 8):

$$
\begin{gathered}
\mathrm{U}^{\mathfrak{s}}=\operatorname{Dropout}\left(\sigma\left(\overrightarrow{\mathfrak{s}} \cdot \mathrm{W}_{1}^{\mathfrak{s}}\right)\right) \mathrm{W}_{2}^{\mathfrak{s}} \\
\mathrm{Z}^{\mathfrak{s}}=\operatorname{Dropout}\left(\sigma\left(\mathrm{U}^{\mathfrak{s}}\right)\right) \\
\Pi(. \mid \overrightarrow{\mathfrak{s}}, \mathcal{A}(\mathrm{u}))=\operatorname{softmax}\left(\mathcal{A}(\mathrm{u}) \odot\left(\mathrm{Z}^{\mathfrak{s}} \cdot \mathrm{W}^{\pi}\right)\right) \\
\Phi(\overrightarrow{\mathfrak{s}})=\mathrm{Z}^{\mathfrak{s}} \cdot \mathrm{W}^{\phi}
\end{gathered}
$$

Where:

- $\mathcal{A}(\mathrm{u})$, is the given binary-encoding-based action matrix of a given user $(\mathrm{u})$.

- $\mathrm{Z}^{\mathfrak{5}}$, is the embedded latent feature matrix of a given state $(\mathfrak{s})$.

- $\overrightarrow{\mathfrak{s}}$, is the latent embedding vector of a specific state ( $\mathfrak{s})$ which is composed by concatenating the embedding vectors of the target user $\left(\overrightarrow{\mathrm{e}_{\mathrm{u}}}\right)$, current visited entity $\left(\overrightarrow{\mathrm{e}_{\mathrm{e}}} \overrightarrow{\mathrm{t}}\right)$ and the historical data $\left(\overrightarrow{\mathrm{e}_{\mathrm{h}} \mathrm{t}}\right)$, denoted as: $\left[\overrightarrow{\mathrm{e}_{\mathrm{u}}}, \overrightarrow{\mathrm{e}_{\mathrm{e}}^{\mathrm{t}}}, \overrightarrow{\mathrm{e}_{\mathrm{h}^{\mathrm{t}}}}\right]$.

Finally, for these proposed policy and value networks, we use stochastic gradient descent (SGD) to jointly optimized the model's parameters, denoted as: $\Theta=\left\{\mathrm{U}^{\mathfrak{5}}, \mathrm{Z}^{\mathfrak{5}}, \mathrm{W}^{\pi}, \mathrm{W}^{\Phi}\right\}$ with the policy gradient upon the loss function of overall given network architectures, as: $\mathcal{L}(\Theta)$, denoted as: $\nabla_{\Theta} \mathcal{L}(\Theta)$ is calculated as the following (as shown in equation 9 and equation 10):

$$
\begin{gathered}
\nabla_{\Theta} \mathcal{L}(\Theta)=\mathbb{E}_{\Phi}\left[\nabla_{\Theta} \cdot \log \Pi(. \mid \overrightarrow{\mathfrak{s}}, \mathcal{A}(\mathrm{u})) \cdot(\mathcal{D}-\Phi(\overrightarrow{\mathfrak{s}}))\right] \\
\Theta_{\text {update }}=\Theta-\eta \nabla_{\Theta} \mathcal{L}(\Theta)
\end{gathered}
$$

Where:

- $\eta$, is the learning rate for updating parameters $(\Theta)$ of given proposed networks via SGD.

- $\mathcal{D}$, is the discounted cumulative reward from the current state $(\mathfrak{s})$ to the terminal state $\left(\mathfrak{S}_{\mathfrak{T}}\right)$.

After the training process of the designed policy network is done, we use the value network to acquire candidate items for each user via generated paths. For the output of each user, we receive a set of $k$-hop candidate paths with the corresponding path-based generative probability and the terminal reward, denoted as: $q_{\mathfrak{I}}$ and $\mathfrak{r}_{\mathfrak{T}}$, respectively. Then, we sort the generated candidate paths following the terminal reward $\mathfrak{r}_{\mathfrak{T}}$ to achieve the top recommended items for each user in a given $\mathrm{KG}$. 


\section{EXPERIMENTS \& DISCUSSIONS}

In this section, we present extensive experiments on real-world recommendation datasets to demonstrate the effectiveness of our proposed HINRL4Rec model in compare with recent recommendation baselines. The experimental outputs demonstrate that our proposed integrated HIN embedding with policy-guided searching mechanism via reinforcement learning approach significantly outperforms recent state-of-the-art methods.

\subsection{Dataset usage and experimental setups}

\subsubsection{Dataset detailed descriptions}

To evaluate the effectiveness of different recommendation methods, we implemented baselines to solve the top-N explainable recommendation task in multiple standard datasets, which are:

- MovieLens: is considered as the classical dataset for evaluating the recommendation technique. MovieLens is provided by the GroupLens with multiple size-varied versions (100K, 1M, 20M, etc.). For experiment in this paper, we mainly used the MovieLens $100 \mathrm{~K}$ version which contains about $100 \mathrm{~K}$ ratings/reviews of $>900$ users for a list of $1,6 \mathrm{~K}$ movies. Beside user and movie entities, the MovieLens dataset also contains other entity's types, such as: 18 movie's genres and 21 user's occupations. The full dataset of MovieLens100K can be download at this repository ${ }^{[1]}$. For the overviews/descriptions and related metadata of movies in this dataset, we downloaded at this repository ${ }^{[2]}$.

- Amazon-Beauty: includes $>250 \mathrm{~K}$ user-item review/rating relationships which are collected from Amazon e-commerce platform is provided by provided by McAuley J. and Leskovec J. [30]. In general, all Amazon-related datasets also contain other types of entities such as: categories (can be downloaded at this repository ${ }^{[3]}$ ) and brands (can be downloaded at this repository ${ }^{[4]}$ ) of reviewed items/products. Moreover, the full-textual descriptions of products/items these datasets can be achieved at this repository ${ }^{[5]}$.

- Amazon-Baby: contains about $>184 \mathrm{~K}$ ratings/reviews about mom/baby related products.

- Amazon-Book: is a large-scale review dataset which contains >12M ratings/reviews from users for related book products.

Textual pre-processing steps \& representation learning. For the textual data related product/item entities, we apply simple pre-processing steps such as: word tokenizing, sentence splitting and word stemming by using the Stanford CoreNLP library ${ }^{[6]}$. Then, to learn the latent representations of these textual data, we apply the default pre-trained BERT model.

\subsubsection{Experimental setups and evaluation metric usage}

To evaluate the accuracy performance of our proposed HIN4Rec model as well as other comparative methods for top- $\mathrm{N}$ recommendation task in different datasets, we use three main evaluation metrics which are: normalized Discounted Cumulative Gain (nDCG), Recall and HitRatio which are similar to evaluation strategy of previous works [3] [31]. As the top-N recommendation task, for all recommendation models, we trained them with the training set and used the trained model to predict the top@k items for each user and validate the recommended product/item sets against the training set in terms of $\mathrm{nDCG}$, Recall and HitRatio metrics. For the MovieLens100K dataset, we used the same training/testing sets in the standard

\footnotetext{
${ }^{[1]}$ MovieLens100K dataset: https://grouplens.org/datasets/movielens/100k/

[2] Metadata and overviews/descriptions of MovieLens100K dataset: https://www.kaggle.com/rounakbanik/the$\underline{\text { movies-dataset }}$

[3] Amazon product's categories: http://snap.stanford.edu/data/amazon/categories.txt.gz

${ }^{[4]}$ Amazon product's brands: http://snap.stanford.edu/data/amazon/brands.txt.gz

${ }^{[5]}$ Amazon product's descriptions: http://snap.stanford.edu/data/amazon/descriptions.txt.gz

${ }^{[6]}$ Stanford CoreNLP library: https://stanfordnlp.github.io/CoreNLP/
} 
version. For Amazon related datasets (Beauty, Baby and Book), we applied the same splitting rules (70\% - training / 30\% - testing) which are also applied in previous works [1] [3] [31]. In more details, for each dataset, we randomly divide the it into two parts: $70 \%$ for model training and the left one is used for validation/testing. We repeated the experiments for each split 10 times and reported the average results as the final outputs. Table 2 shows default configurations of our proposed HINRL4Rec model for all experiments in this paper. We implemented our proposed HINRL4Rec model in Python programming language.

Table 2. Experimental configurations for our proposed HINRLARec model

\begin{tabular}{|l|c|}
\hline \multicolumn{1}{|c|}{ Model's parameter } & Value \\
\hline $\begin{array}{l}\text { Default dimensional size of the textual } \\
\text { embedding vector via BERT and Bi-LSTM. }\end{array}$ & 300 \\
\hline $\begin{array}{l}\text { Default number of hidden states for Bi-LSTM } \\
\text { encoders }(h) \text {. }\end{array}$ & 150 \\
\hline $\begin{array}{l}\text { Default dimensional size meta-path-based } \\
\text { embedding vector }(d) \text {. }\end{array}$ & 300 \\
\hline $\begin{array}{l}\text { Default number of training epochs for } \\
\text { HIN/knowledge graph embedding process. }\end{array}$ & 70 \\
\hline $\begin{array}{l}\text { Default of meta-paths which are used for } \\
\text { MovieLens dataset. }\end{array}$ & $\begin{array}{c}\text { U[user]-O[occupation]-U } \\
\text { U-M[movie]-U } \\
\text { M-G[genres]-M }\end{array}$ \\
\hline $\begin{array}{l}\text { U[user]-P[product]-U } \\
\text { P-U-P }\end{array}$ \\
$\begin{array}{l}\text { Amazon-related datasets (Beauty, Baby and } \\
\text { Book). }\end{array}$ & $\begin{array}{c}\text { P-P (related/also-bought) } \\
\text { P-C[category]-P } \\
\text { P-B[brand]-P }\end{array}$ \\
\hline
\end{tabular}

\subsubsection{Comparative baselines}

In order to prove the effectiveness of our ideas in the proposed HINRL4Rec model, we also implemented several well-known baselines for solving the personalized recommendation task, which are:

- DeepCoNN [32] (or Deep Cooperative Neural Networks): is a recent well-known deep cooperative based approach for recommendation. In the proposed DeepCoNN model, Zheng, L. et al. proposed a novel deep convolutional network-based architecture for effectively recommendation-aware modeling review textual data as well as encoding the user-item interaction data to facilitate the personalized recommendation task. Experimental results which are shown in the original works demonstrate the outperformances of the proposed DeepCoNN model in comparing with recent personalized recommendation techniques. The official source code of DeepCoNN model can be downloaded at this GitHub repository ${ }^{[7]}$.

- TransRec [33]: enables to model the complex user-item interaction as well as sequential pattens of item-item for applying in the context of explainable personalized recommendation task. In the proposed TransRec model, He, R. et al. leverage the performance of sequential recommendation by invoking the translation-based representation learning mechanism. The principal embedding strategy of TransRec model is to jointly transform both user and item entities into the shared representation spaces for facilitating the sequential recommended item extraction. The official source code for the TransRec model from He, R. et al. in $\mathrm{C} / \mathrm{C}++$ language can be download at this repository ${ }^{[8]}$.

${ }^{[7]}$ DeepCoNN (Python): https://github.com/chenchongthu/DeepCoNN

${ }^{[8]}$ TransRec (C/C++): https://sites.google.com/view/ruining-he/ 
- JRL [31]: is a recent state-of-the-art top-N-based recommendation approach which is considered as one of the earliest models for explainable recommendation task. In JRL model, Zhang, Y. et al. proposed a novel technique of combined neural-network-based representation learning technique to learn multi-typed information (e.g., images, texts, etc.) besides the user-item interaction data. The JRL adopts the multiple deep representation learning architecture to effectively learn latent embedding vectors of user and item entities which are then used to facilitate the recommendation task. The official source code for JRL model from Zhang, Y. et al. in Python can be downloaded at this GitHub repository ${ }^{[9]}$.

- HERec [1]: is also known as the first integrated heterogeneous network embedding approach for personalized recommendation problem. In HERec model, Shi, C. et al. proposed a novel fusion technique to merge multi-typed meta-path-based representations of user and item entities in a context of heterogeneous network/knowledge graph into the unified embedding spaces which are then used to facilitate the matrix factorization process for recommendation. Through extensive experiments, HERec model demonstrated remarkable outperformances for personalized recommendation task, comparing with recent baselines in multiple real-world datasets. The official source code for HERec model can be achieved at this GitHub repository ${ }^{[10]}$.

- PGPR [3]: is the recent state-of-the-art explainable recommendation model which is also considered as the main competitor of our proposed HINRL4Rec model in this paper. The PGPR applies the power of reinforcement learning approach for conducting the policy-guided semantic user-item path searching. The PGPR takes advantages of KG embedding via TransE and policy network/value mechanism of reinforcement learning to facilitate the recommendation-driven explainable path-based reasoning process. Moreover, in the proposed PGPR, Xian, Y. et al. proposed a novel user-conditional action pruning strategy to optimize the process of generating reasoning path and speed-up the model's training process. The official source code of PGPR model from Xian, Y. et al. can be achieved at this GitHub repository ${ }^{[11]}$.

All the listed above comparative models are implemented with the same experimental configurations which are listed in Table 2. For the unique configurations of each model, we used the same configurations which make the given model achieves the highest performance as described in the original work.

\subsection{Experimental results \& discussions}

\subsubsection{Recommendation task with different models}

In this section, we conduct the top@ 10 recommendation task by using different recommendation baselines and report the accuracy outputs for top-recommended products of each user in terms of nDCG, Recall and HitRatio metrics within different standard datasets, including: MovieLens, Amazon-Beauty, Amazon-Baby and Amazon-Book. Table 3, Table 4, Table 5 and Table 6 show the experimental outputs for the top@ 10 recommendation task in MovieLens, Amazon-Beauty, Amazon-Baby and Amazon-Book datasets, respectively.

Table 3. Accuracy outputs for top@ 10 recommendation task via different methods in terms of nDCG, Recall and HitRatio evaluation metrics in MovieLens dataset

\begin{tabular}{|l|c|c|c|c|c|c|}
\hline \multicolumn{1}{|c|}{ Model } & \multicolumn{2}{c|}{ nDCG - top@ 10 } & \multicolumn{2}{c|}{ Recall - top@ 10 } & \multicolumn{2}{c|}{ HitRatio - top@ 10 } \\
\hline DeepCoNN & \multicolumn{2}{|c|}{0.03982} & \multicolumn{2}{c|}{0.04792} & \multicolumn{2}{c|}{0.07782} \\
\hline TransRec & 0.04212 & $\uparrow 0.055$ & 0.04891 & $\uparrow 0.020$ & 0.09281 & $\uparrow 0.162$ \\
\hline JRL & 0.04891 & $\uparrow 0.186$ & 0.05891 & $\uparrow 0.187$ & 0.10921 & $\uparrow 0.287$ \\
\hline HERec & 0.05129 & $\uparrow 0.224$ & 0.07329 & $\uparrow 0.346$ & 0.11924 & $\uparrow 0.347$ \\
\hline
\end{tabular}

${ }^{[9]}$ JRL (Python): https://github.com/QingyaoAi/Joint-Representation-Learning-for-Top-N-Recommendation

${ }^{[10]}$ HERec (Python): https://github.com/librahu/HERec

${ }^{[11]}$ PGPR (Python): https://github.com/orcax/PGPR 


\begin{tabular}{|l|l|l|l|l|l|l|}
\hline PGPR & 0.05382 & $\uparrow 0.260$ & 0.07201 & $\uparrow 0.335$ & 0.12821 & $\uparrow 0.393$ \\
\hline HINRL4Rec & $\mathbf{0 . 0 5 7 8 1}$ & $\uparrow 0.311$ & $\mathbf{0 . 0 7 8 9 2}$ & $\uparrow 0.393$ & $\mathbf{0 . 1 4 9 3 2}$ & $\uparrow 0.479$ \\
\hline
\end{tabular}

Table 4. Accuracy outputs for top@10 recommendation task via different methods in terms of nDCG, Recall and HitRatio evaluation metrics in Amazon-Beauty dataset

\begin{tabular}{|l|l|l|l|l|l|l|}
\hline \multicolumn{1}{|c|}{ Model } & \multicolumn{2}{c|}{ nDCG - top@ 10 } & \multicolumn{2}{c|}{ Recall - top@ 10 } & \multicolumn{2}{c|}{ HitRatio - top@ 10 } \\
\hline DeepCoNN & \multicolumn{2}{|c|}{0.03921} & \multicolumn{2}{c|}{0.05692} & \multicolumn{2}{c|}{0.10923} \\
\hline TransRec & 0.03792 & $\downarrow 0.034$ & 0.05227 & $\downarrow 0.089$ & 0.09823 & $\downarrow 0.112$ \\
\hline JRL & 0.03829 & $\downarrow 0.024$ & 0.07213 & $\uparrow 0.211$ & 0.11294 & $\uparrow 0.033$ \\
\hline HERec & 0.04812 & $\uparrow 0.185$ & 0.08872 & $\uparrow 0.358$ & 0.14912 & $\uparrow 0.268$ \\
\hline PGPR & 0.05408 & $\uparrow 0.275$ & 0.08561 & $\uparrow 0.335$ & 0.14721 & $\uparrow 0.258$ \\
\hline HINRL4Rec & $\mathbf{0 . 0 5 5 2 1}$ & $\uparrow 0.290$ & $\mathbf{0 . 0 9 1 3 2}$ & $\uparrow 0.377$ & $\mathbf{0 . 1 5 0 1 1}$ & $\uparrow 0.272$ \\
\hline
\end{tabular}

Table 5. Accuracy outputs for top@10 recommendation task via different methods in terms of nDCG, Recall and HitRatio evaluation metrics in Amazon-Baby dataset

\begin{tabular}{|l|l|l|l|l|l|l|}
\hline & \multicolumn{2}{|c|}{ nDCG - top@ 10 } & \multicolumn{2}{c|}{ Recall - top@ 10 } & \multicolumn{2}{c|}{ HitRatio - top@ 10 } \\
\hline DeepCoNN & \multicolumn{2}{|c|}{0.04821} & \multicolumn{2}{c|}{0.05692} & \multicolumn{2}{c|}{0.12891} \\
\hline TransRec & 0.05192 & $\uparrow 0.071$ & 0.06672 & $\uparrow 0.147$ & 0.13781 & $\uparrow 0.065$ \\
\hline JRL & 0.05267 & $\uparrow 0.085$ & 0.06592 & $\uparrow 0.137$ & 0.14812 & $\uparrow 0.130$ \\
\hline HERec & 0.06183 & $\uparrow 0.220$ & 0.07192 & $\uparrow 0.209$ & 0.16827 & $\uparrow 0.234$ \\
\hline PGPR & 0.06421 & $\uparrow 0.249$ & 0.07492 & $\uparrow 0.240$ & 0.17472 & $\uparrow 0.262$ \\
\hline HINRL4Rec & $\mathbf{0 . 0 6 4 8 1}$ & $\uparrow 0.256$ & $\mathbf{0 . 0 7 5 8 2}$ & $\uparrow 0.249$ & $\mathbf{0 . 1 7 8 2 1}$ & $\uparrow 0.277$ \\
\hline
\end{tabular}

Table 6. Accuracy outputs for top@10 recommendation task via different methods in terms of nDCG, Recall and HitRatio evaluation metrics in Amazon-Book dataset

\begin{tabular}{|l|c|c|c|c|c|c|}
\hline & \multicolumn{2}{|c|}{ nDCG - top@ 10 } & \multicolumn{2}{c|}{ Recall - top@ 10 } & \multicolumn{2}{c|}{ HitRatio - top@ 10 } \\
\hline DeepCoNN & \multicolumn{2}{|c|}{0.03288} & \multicolumn{2}{c|}{0.048825} & \multicolumn{2}{c|}{0.09823} \\
\hline TransRec & 0.03129 & $\downarrow 0.051$ & 0.04782 & $\downarrow 0.021$ & 0.09782 & $\downarrow 0.004$ \\
\hline JRL & 0.03482 & $\uparrow 0.056$ & 0.05182 & $\uparrow 0.058$ & 0.10928 & $\uparrow 0.101$ \\
\hline HERec & 0.04192 & $\uparrow 0.216$ & 0.06489 & $\uparrow 0.248$ & 0.13982 & $\uparrow 0.297$ \\
\hline PGPR & 0.04082 & $\uparrow 0.195$ & 0.06402 & $\uparrow 0.237$ & 0.14031 & $\uparrow 0.300$ \\
\hline HINRL4Rec & $\mathbf{0 . 0 4 3 2 1}$ & $\uparrow 0.239$ & $\mathbf{0 . 0 6 5 7 6}$ & $\uparrow 0.258$ & $\mathbf{0 . 1 4 7 2 3}$ & $\uparrow 0.333$ \\
\hline
\end{tabular}

Experimental outputs demonstrate that our proposed HINRL4Rec model outperforms most of recent recommendation baselines. In more details, for the classical recommendation dataset such as MovieLens, the HINRL4Rec model achieves better performance than PGPR $(7.41 \%, 9.59 \%$ and $16.45 \%)$, HERec $(12.71 \%, 7.68 \%$ and $25.22 \%)$, JRL $(18.19 \%, 33.97 \%$ and $36.72 \%)$, TransRec $(37.23 \%, 61.35 \%$ and $60.88 \%)$ and DeepCoNN $(45.17 \%, 64.7 \%$ and $91.87 \%)$ in terms of top@ 10 nDCG, Recall and HitRatio metrics, respectively. Similar to that in Amazon-related datasets, our proposed HINRL4Rec also significantly outperforms averagely $26.53 \%, 35.39 \%$ and $38.04 \%$ in comparing with JRL, TransRec and DeepCoNN in terms of top@ $10 \mathrm{nDCG}$, metrics. For our main competitors in this paper which are HERec and PGPR, our proposed model also slightly achieves better accuracy performance approximately $3.81 \%$ and $8.8 \%$ in terms of top@ 10 nDCG for all three datasets, including: Amazon-Beauty, Amazon-Baby and Amazon-Book datasets. 

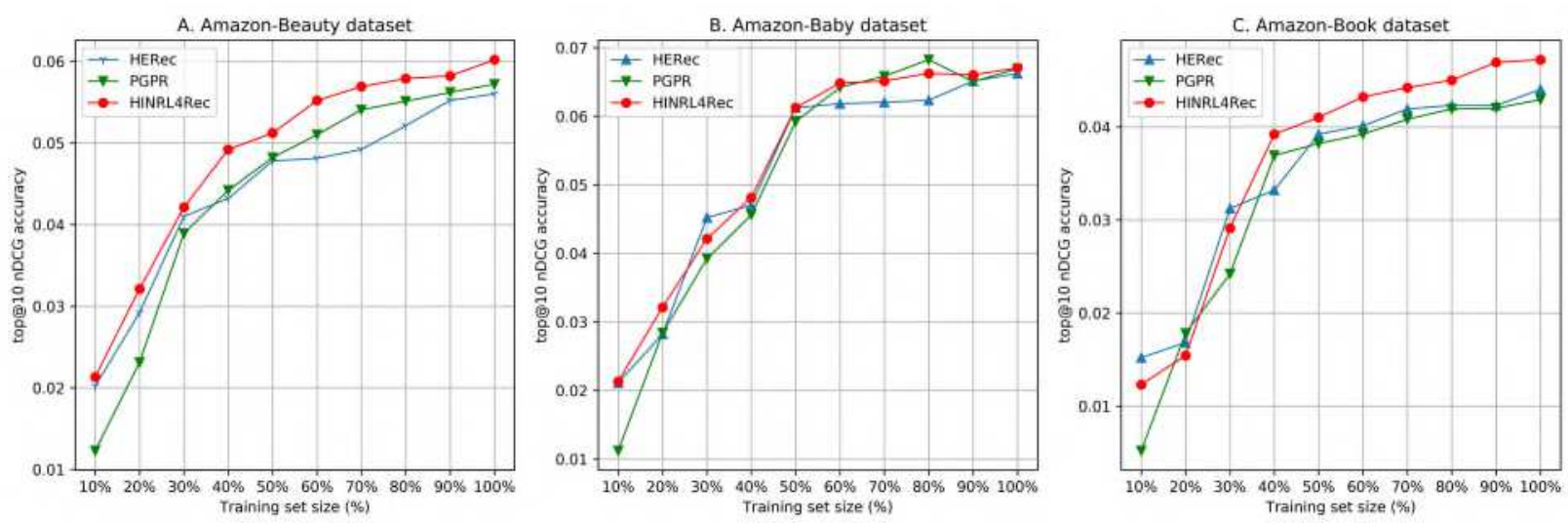

Figure 3. Experimental evaluations on the stability of recommendation models in Amazon-related datasets with different sizes of training set $(\%)$

Model' stability evaluation. In this experiment, we tried to evaluate and compare the stability of our proposed HINRL4Rec model with our main competitors which are HERec and PGPR in the context of sizevaried training set. To do this, we varied the training set size (\%) of Amazon-related datasets from $10 \%$ to $100 \%$ (increases 10 for each step), then reported the changes of overall accuracy performance of different recommendation techniques, including: HERec, PGPR and HINRL4Rec in terms of top@10 nDCG evaluation metric.

Experimental outputs in Figure 3 demonstrates that all implemented recommendation baselines are quite stable in all Amazon-Beauty, Amazon-Baby and Amazon-Book datasets, where the top@ 10 nDCG scores increase accordingly with the sizes of training set (\%). For thorough evaluations on the large-scale and dense datasets like as Amazon-Book, our proposed HINRL4Rec is considered more stable than HERec and PGPR with the average increase of approximately $18.63 \%$ for different sizes of the training set. These experimental outputs prove that our proposed HINRL4Rec could be flexible and stable for implementing in the context of size-varied dataset.

\subsubsection{Studies on model's parameter sensitivity}

As mainly a heterogeneous embedding based approach which taking advantages of multiple latent representations of both textual and structural meta-path-based embedding techniques for leveraging the performance of personalized recommendation task, we furtherly conducted extensive studies on evaluating on the influences of critical model's pre-defined parameters such as dimensional embedding sizes, denoted as: $(d)$ of both textual and meta-path-based embedding vectors for target-typed entities (e.g. users and products/items/movies), number of hidden states/LSTM-based cells, denoted as (h) of Bi-LSTM encoding mechanisms which are used for learning the sequential representations of associated textual data of items (products/items/movies) as well as the default setup training epochs for our proposed HINRL4Rec to reach the highest performance. For the experiments on evaluation the influences of $(d)$ and $(h)$ parameters on overall our proposed HINRL4Rec's model accuracy for the recommendation task, we varied the values of $(d)$ and $(h)$ parameters in range [0-400] (50 for each increasing step) and [0-300] (50 for each increasing step), respectively, and reported the changes in accuracy performances of our proposed HINRL4Rec in terms of nDCG metric. Similar to that, for the configured number of model training epochs, we also varied this parameter's value in range [10-100] (increases 10 for each step) and investigated the fluctuations on the overall model accuracy in terms of nDCG metric. For all experiments in this section, we all used the Amazon-related datasets (Beauty, Baby and Book). Experimental outputs in Figure 4-A and Figure 4-B show the fact is that our proposed HINRL4Rec model is quite insensitive with both $(\mathrm{d})$ and $(\mathrm{h})$ parameters. In more details, for different datasets, our proposed model gradually achieves the highest performance accordingly with the stably increases of (d) and (h) parameters, for small datasets such as: Amazon-Beauty and Amazon-Baby, it reaches the highest performances with the values of (d) and (h) parameters are $>200$ 
and 50, respectively. For large-scale dataset such as Amazon-Book, the model gains the high performances with the values of (d) and (h) parameters approximately over 300 and 50, respectively. Similar to that with the experiments with number of training epoch parameter, depending on the size of dataset, our proposed HINRL4Rec model need different number of training epochs to reach the convergent point, for AmazonBeauty and Amazon-Baby are > 60 whereas over 70 for the Amazon-Book dataset.
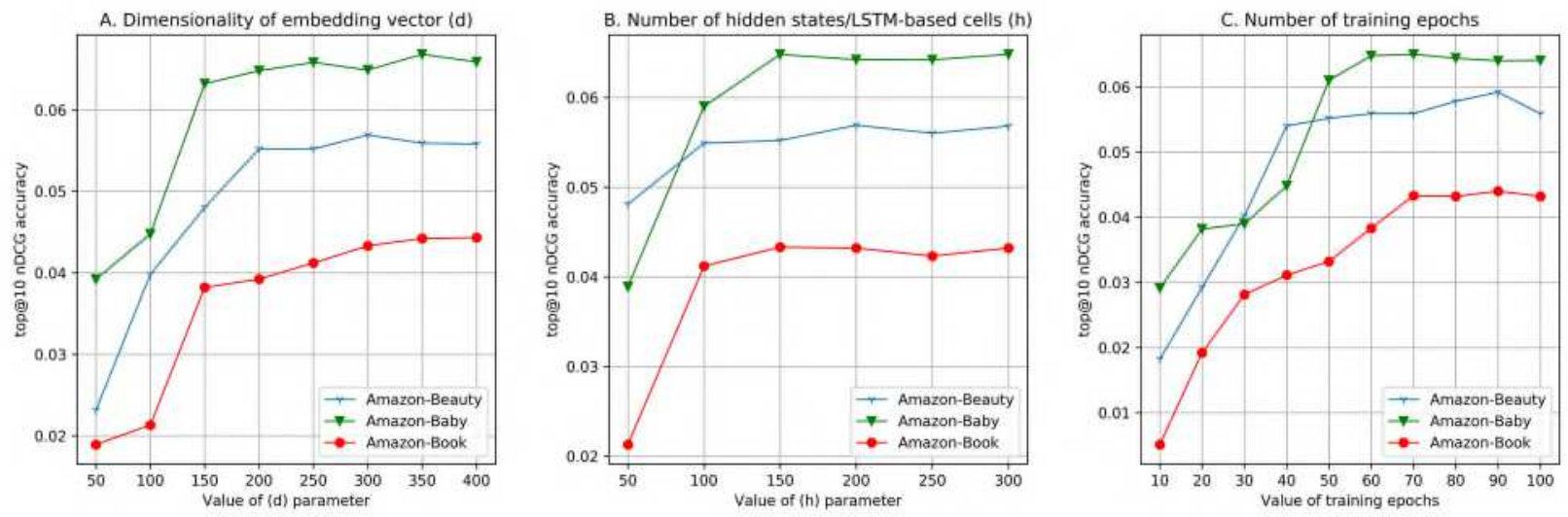

Figure 4. Experimental evaluations on the parameter sensitivity of our proposed HINRLARec model in different Amazon-related datasets

\section{CONCLUSIONS \& FUTURE WORKS}

To sum up, in this paper we propose a novel approach of integrated heterogeneous network embedding with the policy-guided path-based searching mechanism via reinforcement learning, called as HINRL4Rec for leveraging the performance of explainable personalized recommendation task. In our proposed HINRL4Rec model, we firstly proposed a jointly textual and meta-path-based embedding technique called as MultMP-KG2Vec to effectively learn the rich semantic representations of target-typed entities in a given knowledge graph. Then, the achieved meta-path-varied representations of target-typed entities are merged into the unified target embedding spaces by utilizing multiple fusion mechanism which are then used to facilitate the policy network training process. The trained policy-driven path searching model via reinforcement learning approach is then used to generate meaningful user-item inter-connecting paths for the explainable recommendation outputs. Extensive experiments in standard datasets demonstrate the outperformances of our proposed HINRL4Rec model in comparing with recent state-of-the-art baselines. For future improvements, we would like to extend our proposed HINRL4Rec model for handling the friendship recommendation task in social networks as much richer contextual information than knowledge graphs for product/movie recommendations. Moreover, the proposed HINRL4Rec is also improved to be capable for capturing the temporal information of heterogeneous networks in the context of dynamic network evolution over the time.

\section{DEClarations}

This research is funded by Thu Dau Mot University, Binh Duong, Vietnam.

\section{ACKNOWLEDGEMENT}

This research is funded by Thu Dau Mot University, Binh Duong, Vietnam.

\section{CONFLICT OF INTEREST}

This research is funded by Thu Dau Mot University, Binh Duong, Vietnam. 


\section{REFERENCES}

[1] Zhang, S., Yao, L., Sun, A., \& Tay, Y., "Deep learning based recommender system: A survey and new perspectives," ACM Computing Surveys (CSUR), vol. 52, no. 1, pp. 1-38, 2019.

[2] Shi, C., Hu, B., Zhao, W. X., \& Philip, S. Y., "Heterogeneous information network embedding for recommendation," IEEE Transactions on Knowledge and Data Engineering, vol. 31, no. 2, pp. 357-370, 2018.

[3] Zhao, Z., Zhang, X., Zhou, H., Li, C., Gong, M., \& Wang, Y., "HetNERec: Heterogeneous network embedding based recommendation," Knowledge-Based Systems, vol. 204, p. 106218, 2020.

[4] Xian, Y., Fu, Z., Muthukrishnan, S., De Melo, G., \& Zhang, Y., "Reinforcement knowledge graph reasoning for explainable recommendation," in Proceedings of the 42nd international ACM SIGIR conference on research and development in information retrieval, 2019.

[5] Lei, W., Zhang, G., He, X., Miao, Y., Wang, X., Chen, L., \& Chua, T. S., "Interactive path reasoning on graph for conversational recommendation," in Proceedings of the 26th ACM SIGKDD International Conference on Knowledge Discovery \& Data Mining, 2020.

[6] Bordes, A., Usunier, N., Garcia-Duran, A., Weston, J., \& Yakhnenko, O., "Translating embeddings for modeling multirelational data," Advances in neural information processing systems, pp. 2787-2795, 2013.

[7] Wang, Z., Zhang, J., Feng, J., \& Chen, Z., "Knowledge graph embedding by translating on hyperplanes," in Twenty-Eighth AAAI conference on artificial intelligence, 2014.

[8] Lin, Y., Liu, Z., Sun, M., Liu, Y., \& Zhu, X., "Learning entity and relation embeddings for knowledge graph completion," in Twenty-ninth AAAI conference on artificial intelligence, 2015.

[9] Perozzi, B., Al-Rfou, R., \& Skiena, S., "Deepwalk: Online learning of social representations," in Proceedings of the 20th ACM SIGKDD international conference on Knowledge discovery and data mining, 2014.

[10] Tang, J., Qu, M., Wang, M., Zhang, M., Yan, J., \& Mei, Q., "Line: Large-scale information network embedding," in Proceedings of the 24th international conference on world wide web, 2015.

[11] Grover, A., \& Leskovec, J., "node2vec: Scalable feature learning for networks," in Proceedings of the 22nd ACM SIGKDD international conference on Knowledge discovery and data mining, 2016.

[12] Sun, Y., Han, J., Yan, X., Yu, P. S., \& Wu, T., "Pathsim: Meta path-based top-k similarity search in heterogeneous information networks," in Proceedings of the VLDB Endowment, 2011.

[13] Shi, C., Li, Y., Zhang, J., Sun, Y., \& Philip, S. Y., "A survey of heterogeneous information network analysis," IEEE Transactions on Knowledge and Data Engineering, vol. 29, no. 1, pp. 17-37, 2016.

[14] Ai, Q., Azizi, V., Chen, X., \& Zhang, Y., "Learning heterogeneous knowledge base embeddings for explainable recommendation," Algorithms, vol. 11, no. 9, p. 137, 2018.

[15] Wang, X., Xu, Y., He, X., Cao, Y., Wang, M., \& Chua, T. S., "Reinforced negative sampling over knowledge graph for recommendation," in Proceedings of The Web Conference 2020, 2020.

[16] Devlin, J., Chang, M. W., Lee, K., \& Toutanova, K., "Bert: Pre-training of deep bidirectional transformers for language understanding," in Proceedings of the 2019 Conference of the North American Chapter of the Association for Computational Linguistics: Human Language Technologies, 2019.

[17] Xie, H., Li, Q., Mao, X., Li, X., Cai, Y., \& Rao, Y., "Community-aware user profile enrichment in folksonomy," Neural Networks, vol. 58, pp. 111-121, 2014.

[18] Liang, D., Altosaar, J., Charlin, L., \& Blei, D. M., "Factorization meets the item embedding: Regularizing matrix factorization with item co-occurrence," in Proceedings of the 10th ACM conference on recommender systems, 2016. 
[19] Cao, D., Nie, L., He, X., Wei, X., Zhu, S., \& Chua, T. S., "Embedding factorization models for jointly recommending items and user generated lists," in Proceedings of the 40th International ACM SIGIR Conference on Research and Development in Information Retrieval, 2017.

[20] $\mathrm{Yu}, \mathrm{X}$. et al., "Personalized entity recommendation: A heterogeneous information network approach," in Proceedings of the 7th ACM international conference on Web search and data mining, 2014.

[21] Luo, C., Pang, W., Wang, Z., \& Lin, C., "Hete-cf: Social-based collaborative filtering recommendation using heterogeneous relations," in 2014 IEEE International Conference on Data Mining, 2014.

[22] Shi, C., Zhang, Z., Luo, P., Yu, P. S., Yue, Y., \& Wu, B., "Semantic path based personalized recommendation on weighted heterogeneous information networks," in Proceedings of the 24th ACM International on Conference on Information and Knowledge Management, 2015.

[23] Dong, Y., Chawla, N. V., \& Swami, A., "metapath2vec: Scalable representation learning for heterogeneous networks," in Proceedings of the 23rd ACM SIGKDD international conference on knowledge discovery and data mining, 2017.

[24] Zhang, D., Yin, J., Zhu, X., \& Zhang, C., "Metagraph2vec: Complex semantic path augmented heterogeneous network embedding," in Pacific-Asia conference on knowledge discovery and data mining, 2018.

[25] Chen, H., Yin, H., Wang, W., Wang, H., Nguyen, Q. V. H., \& Li, X., "PME: projected metric embedding on heterogeneous networks for link prediction," in Proceedings of the 24th ACM SIGKDD International Conference on Knowledge Discovery \& Data Mining, 2018.

[26] Zhang, C., Swami, A., \& Chawla, N. V., "SHNE: Representation learning for semantic-associated heterogeneous networks," in Proceedings of the Twelfth ACM International Conference on Web Search and Data Mining, 2019.

[27] Zhang, Y., \& Chen, X., "Explainable recommendation: A survey and new perspectives," Foundations and Trends ${ }^{\circledR}$ in Information Retrieval, vol. 14, no. 1, pp. 1-101, 2020.

[28] Wang, X., Wang, D., Xu, C., He, X., Cao, Y., \& Chua, T. S., "Explainable reasoning over knowledge graphs for recommendation," in Proceedings of the AAAI Conference on Artificial Intelligence, 2019.

[29] Mikolov, T., Chen, K., Corrado, G., \& Dean, J., "Efficient estimation of word representations in vector space," arXiv preprint arXiv:1301.3781, 2013.

[30] Sutton, R. S., \& Barto, A. G., Reinforcement learning: An introduction, MIT press, 2018.

[31] McAuley, J., \& Leskovec, J., "Hidden factors and hidden topics: understanding rating dimensions with review text," in Proceedings of the 7th ACM conference on Recommender systems, 2013.

[32] Zhang, Y., Ai, Q., Chen, X., \& Croft, W. B., "Joint representation learning for top-n recommendation with heterogeneous information sources," in Proceedings of the 2017 ACM on Conference on Information and Knowledge Management, 2017.

[33] Zheng, L., Noroozi, V., \& Yu, P. S., "Joint deep modeling of users and items using reviews for recommendation," in Proceedings of the tenth ACM international conference on web search and data mining, 2017.

[34] He, R., Kang, W. C., \& McAuley, J., "Translation-based recommendation," in Proceedings of the eleventh ACM conference on recommender systems, 2017. 